\title{
Ever-present Majorana bound state in a generic one-dimensional superconductor with odd number of Fermi surfaces
}

\author{
Maxim Kharitonov, ${ }^{1,2}$ Ewelina M. Hankiewicz, ${ }^{1,3}$ Björn Trauzettel, ${ }^{1,3}$ and F. Sebastian Bergeret ${ }^{2,4}$ \\ ${ }^{1}$ Institute for Theoretical Physics and Astrophysics, University of Würzburg, 97074 Würzburg, Germany \\ ${ }^{2}$ Donostia International Physics Center (DIPC), Manuel de Lardizabal 4, E-20018 San Sebastián, Spain \\ ${ }^{3}$ Würzburg-Dresden Cluster of Excellence ct.qmat, Germany \\ ${ }^{4}$ Centro de Física de Materiales (CFM-MPC), Centro Mixto CSIC-UPV/EHU, Manuel de Lardizabal 5, E-20018 San Sebastián, Spain
}

(Received 17 September 2020; revised 17 August 2021; accepted 10 September 2021; published 26 October 2021)

A quasi-1D superconductor with an odd number of Fermi surfaces is expected to exhibit a nondegenerate Majorana bound state at the Fermi level at its boundary with an insulator (where the latter could be an actual insulator material or vacuum, for a terminated sample). Previous explicit theoretical demonstrations of this property were done for specific microscopic models of the bulk Hamiltonian and, most importantly, of the boundary. In this work, we theoretically demonstrate that this property holds for the whole class of systems, using the symmetry-based formalism of low-energy continuum models and general boundary conditions. We derive the general form of the Bogoliubov-de Gennes low-energy Hamiltonian that is subject only to charge-conjugation symmetry $\mathcal{C}_{+}$of the type $\mathcal{C}_{+}^{2}=+1$ and a few minimal assumptions. Crucially, we also derive the most general form of the boundary conditions describing the boundary with an insulator, subject only to the fundamental principle of the probability-current conservation and $\mathcal{C}_{+}$symmetry. Such normal-reflection boundary conditions do not contain scattering between electrons and holes. We find that for an odd number of Fermi surfaces a Majorana bound state always exists as long as the bulk is in the gapped superconducting state, irrespective of the parameters of the bulk Hamiltonian and boundary conditions. Importantly, our general model includes a possible Fermi-point mismatch, when the two Fermi points are not at exactly opposite momenta, which disfavors superconductivity. We find that the Fermi-point mismatch does not have a direct destructive effect on the Majorana bound state, in the sense that once the bulk gap is opened the bound state is always present.

DOI: 10.1103/PhysRevB.104.134516

\section{INTRODUCTION AND MAIN RESULTS}

Majorana bound states [1-29] in quasi-one-dimensional superconductors have attracted a lot of interest recently, largely due to the prospects of implementing them as the basis for quantum computing. Theoretical predictions for specific systems, such as a quantum wire [3,4] (Fig. 1) or an edge of a quantum spin Hall system [2] (Fig. 2) in proximity to a superconductor, have been made and are being currently experimentally explored [27-29].

A quasi-1D superconductor with an odd number of Fermi surfaces (FSs) (by a Fermi surface in 1D we mean a pair of Fermi points with right- and left-moving electrons, in the geometry of Fig. 3) is expected on topological grounds to exhibit a nondegenerate Majorana bound state at the Fermi level at its boundary with an insulator (where the latter could be an actual insulator material or vacuum, for a terminated sample). Previous explicit theoretical demonstrations [1-15,18-20] of this property were done for specific microscopic models of the bulk Hamiltonian and, most importantly, of the boundary.

In this work, we theoretically demonstrate that this property holds for the whole class of systems, using the symmetry-based formalism of low-energy continuum models and general boundary conditions (BCs). Remarkably, this formalism allows one to study the bound states of topological systems in an explicit and general fashion, while completely bypassing topological arguments, such as appeal to bulkboundary correspondence.

In most of the paper, we perform such analysis for the case of one Fermi surface (1FS) and then generalize this result to the case of an arbitrary odd number of Fermi surfaces.

For the case of 1FS, under the approximation of the linearized normal-state spectrum close to the Fermi points (Fig. 3), we derive the most general form of the single-particle Bogoliubov-de Gennes [30] (BdG) low-energy Hamiltonian that is subject only to charge-conjugation (CC) symmetry $\mathcal{C}_{+}$ of the type $\mathcal{C}_{+}^{2}=+1$. Such system belongs to the symmetry class D [31,32]; physically, this describes spinful superconductors with no other assumed symmetries, in particular, with broken time-reversal (TR) symmetry $\mathcal{T}_{-}, \mathcal{T}_{-}^{2}=-1$.

Crucially, for 1FS, we also derive the most general form of the BCs for this low-energy Hamiltonian, subject only to the fundamental principle of the probability-current conservation [33-43] and to CC symmetry $\mathcal{C}_{+}$. We find that there are two families of such $\mathrm{BCs}$, which we term normal-reflection and Andreev-reflection BCs. In the normal-reflection $\mathrm{BCs}$, there is no scattering between electrons and holes; electrons are reflected as electrons and holes as holes. In the Andreevreflection BCs, there is complete reflection of electrons as holes and vice versa. 

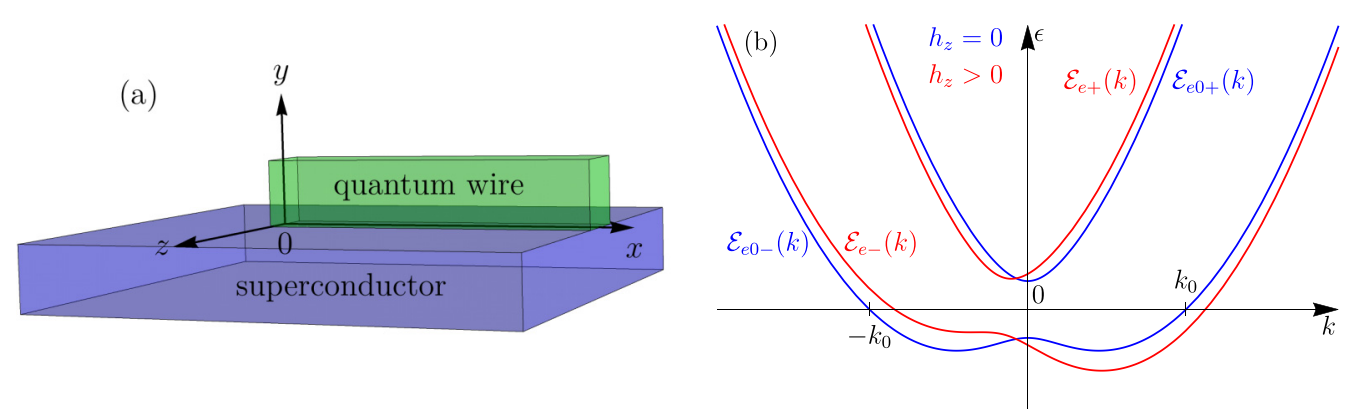

FIG. 1. (a) The system of a quantum wire coupled to a superconductor by the proximity effect $[3,4]$, considered in Sec. VII. The Zeeman field is required to create the regime of 1FS. Spin-orbit interactions are required to induce superconductivity in the wire from the superconductor with spin-singlet pairing. For the Zeeman field in the vertical plane containing the wire axis, the electron system has [45,47,48] an effective time-reversal (TR) symmetry with the operation $\mathcal{T}_{e+}=\Sigma_{z} \mathcal{T}_{e-}, \mathcal{T}_{e+}^{2}=1$, that is the product of the actual TR operation $\mathcal{T}_{e-}, \mathcal{T}_{e-}^{2}=-1$, and reflection $\Sigma_{z}$ along the horizontal direction $z$ perpendicular to the wire [49]. The component $h_{z}$ of the Zeeman field along the $z$ direction breaks this $\mathcal{T}_{e+}$ symmetry and creates a Fermi-point mismatch in the bulk normal-state electron spectrum $\mathcal{E}_{ \pm}(k)$ [Eq. (7.4)] (red), shown in (b). The spectrum $\mathcal{E}_{e 0 \pm}(k)=\mathcal{E}_{e 0 \pm}(-k)$ [Eq. (7.5)] (blue) at $h_{z}=0$ is $k \leftrightarrow-k$ symmetric due to the said $\mathcal{T}_{e+}$ symmetry.

In this work, the physical systems of interest are superconductors interfaced with an insulator. Such boundaries can only be described by normal-reflection BCs. One the other hand, Andreev-reflection BCs cannot be defined in the normal state and thus cannot represent the boundary with an insulator. For this reason, we explore the bound states only for the normalreflection BCs. For the normal-reflection BCs, we find that a single Majorana bound state always exists at the boundary of a half-infinite system (Fig. 4), as long as the bulk is in the gapped superconducting state, irrespective of the parameters of the bulk Hamiltonian and BCs, thereby proving our claim formulated above.

Importantly, our general model includes the possible Fermi-point mismatch (Fig. 3), i.e., the situation when the two Fermi points are not at exactly opposite momenta [20,44], which disfavors superconductivity and introduces a threshold (Figs. 4 and 5) for creating a gapped superconducting state by a coordinate-independent pairing field. We also include the one-harmonic periodic coordinate dependence of the pairing field, which could help mitigate this effect. We find that the Fermi-point mismatch does not have a direct destructive effect on the Majorana bound state, in the sense that once the bulk gap is opened the bound state is always present.

We stress that our approach proves the existence of Majorana bound states generally, since this is demonstrated within the low-energy model of the most general form, constrained only by the CC symmetry and the probability-current conservation principle. The only requirement for the applicability of the low-energy model for 1FS is the smallness of the superconducting pairing field and possible Fermi-point mismatch compared to the nonlinearity scale of the underlying normal-state band (these constraints can also be relaxed by the continuity argument). In this low-energy limit, any microscopic BdG model will reduce to an instance of the derived low-energy model with specific parameters of its Hamiltonian and BCs. We illustrate such systematic procedure of deriving the low-energy model from the microscopic model with two examples: a generalized quantum-wire model (Fig. 1) and a model of the edge of the quantum spin Hall system interfaced with a magnetic material (Fig. 2).

This claim thus holds for any microscopic BdG model, regardless of the structure of its normal-state bulk Hamiltonian, spin structure of the superconducting pairing field, and boundary with an insulator. Our low-energy symmetry-based approach thus proves that the persistence of Majorana bound states is, in fact, the property of the whole class of systems. This is the main difference from the previous theoretical demonstrations of the existence of the Majorana bound states, which were done for specific microscopic models.

The above approach for 1FS allows for a straightforward generalization to an arbitrary number of Fermi surfaces. The only additional assumption we make is to neglect
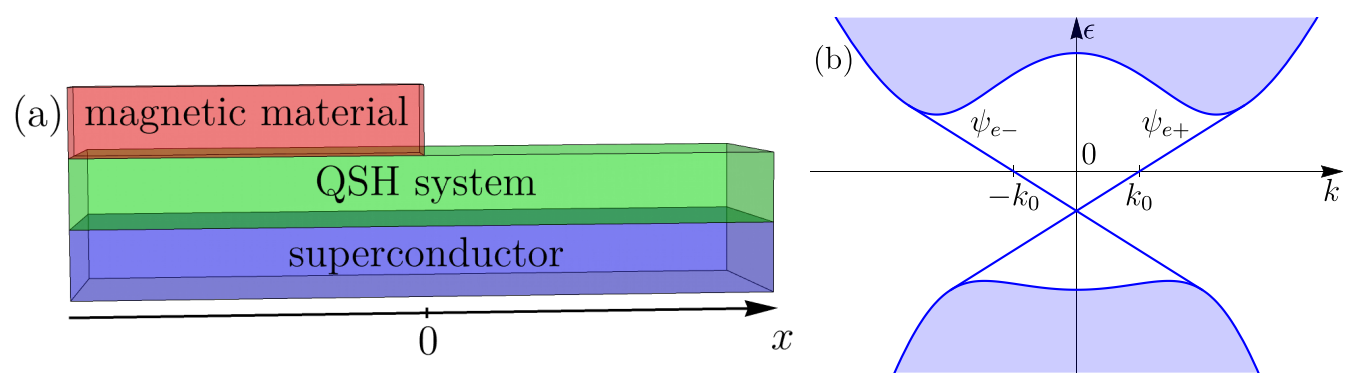

FIG. 2. (a) The edge of a quantum spin Hall system coupled to a superconductor by the proximity effect [2], considered in Sec. VIII. The magnetic material placed in the region $x<0$ breaks the time-reversal symmetry $\mathcal{T}_{e-}, \mathcal{T}_{e-}^{2}=-1$, of the electron system and causes backscattering of the counterpropagating edge states. (b) The schematic normal-state electron spectrum of the system, with counterpropagating edge states and bulk continuum (shaded regions). 


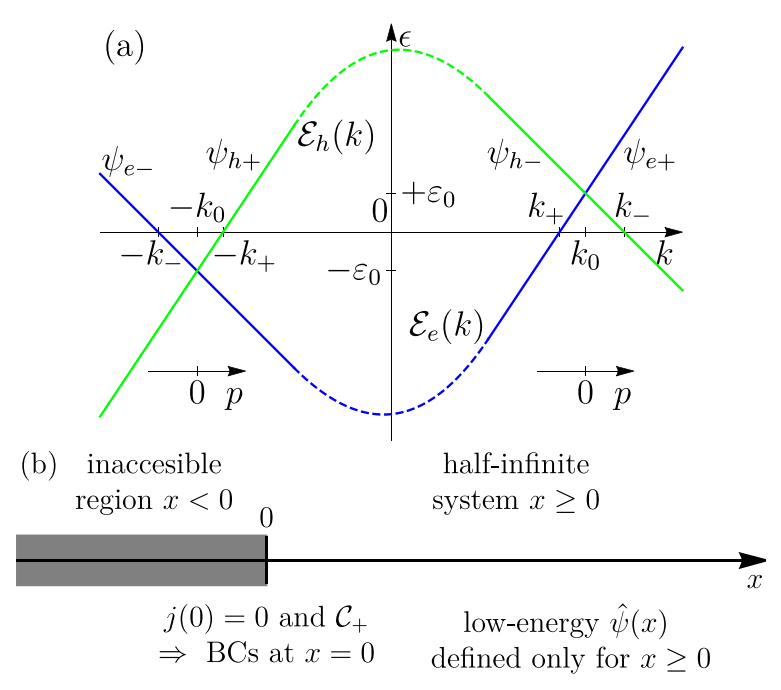

FIG. 3. The origin of the generic low-energy model of a 1D superconductor in the regime of one Fermi surface (1FS). (a) The schematic of the electron $\mathcal{E}_{e}(k)$ (blue) and hole $\mathcal{E}_{h}(k)=-\mathcal{E}_{e}(-k)$ (green) bulk bands in the normal state, in the absence of superconducting pairing. The solid-line parts show regions of the linearized spectrum close to the Fermi level, where the low-energy model [Eqs. (2.2) and (2.11)] applies, with the electron $\psi_{e \pm}(x)$ and hole $\psi_{h \pm}(x)$ components of the $\mathrm{BdG}$ wave function $\hat{\psi}(x)$ [Eq. (2.5)]. Generally, $\mathcal{E}_{e}(k) \neq \mathcal{E}_{e}(-k)$ and a Fermi-point mismatch is present, when the Fermi points $\pm k_{ \pm}$are not at opposite momenta, $k_{+} \neq k_{-}$. In this case, the electron $\mathcal{E}_{e}(k)$ and hole $\mathcal{E}_{h}(k)$ bands cross at opposite momenta $\pm k_{0}$ at finite mismatch energies $\pm \varepsilon_{0}$ [Eq. (2.1)] relative to the Fermi level. (b) Half-infinite low-energy system $x \geqslant 0$ with a boundary $x=0$ used to calculate bound states. The low-energy BdG wave function $\hat{\psi}(x)$ is not defined in the "inaccessible" region $x<0$. Instead, the general boundary conditions [Eqs. (3.5) and (3.6)] at $x=0$ that satisfy only the fundamental probability-current conservation principle [Eq. (3.1)] and charge-conjugation (CC) symmetry $\mathcal{C}_{+}$are imposed (Sec. III, Fig. 6).

superconducting pairing between different Fermi surfaces, which is justified in the low-energy limit when the Fermi surfaces are well separated. For more than 1FS, we derive from the start only the most general form of the normalreflection $\mathrm{BCs}$, in which scattering between electrons and holes is absent, since only such BCs describe the boundary with an insulator. For an odd number of Fermi surfaces, we find that a Majorana bound state exists, irrespective of the parameters of the bulk Hamiltonian and BCs.

Our findings have crucial implications for the stability and persistence of Majorana bound states in real systems and can be used as a general guide for engineering systems that host Majorana bound states: as long as the basic general requirements of creating a system with an odd number of Fermi surfaces and inducing a gapped superconducting state in it are achieved, Majorana bound states are guaranteed to exist.

The paper is organized as follows. All sections, except for Sec. VI, are devoted to the case of 1FS. In Sec. II, we derive the low-energy Hamiltonian of the most general form. In Sec. III, we derive the BCs of the most general form. In Sec. IV, we analyze the Fermi-point mismatch and introduce the coordinate dependence of the pairing field that may help

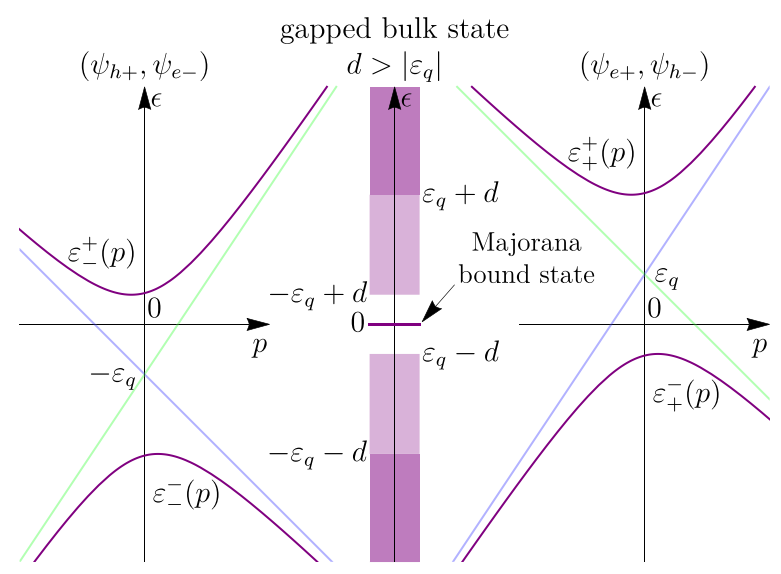

FIG. 4. Ever-present Majorana bound state in the gapped superconducting state of the low-energy model with 1FS [Eqs. (2.11) and (3.5)], the main result of this work. Our model includes the effect of the Fermi-point mismatch (Fig. 3) and the one-harmonic coordinate dependence $\Delta_{q}(x)=\Delta_{0} e^{i q x}$ [Eq. (4.1)] of the pairing field that can help mitigate it. The bulk spectrum (4.6) has individual energy gaps $\left(\varepsilon_{q}-d, \varepsilon_{q}+d\right)$ and $\left(-\varepsilon_{q}-d,-\varepsilon_{q}+d\right)$ around $\pm k_{0}$ momenta, respectively, that open around the modified mismatch energies $\pm \varepsilon_{q}$ for any magnitude $\Delta_{0}$ of the pairing field [Eqs. (4.5) and (4.7)]. For large enough $\Delta_{0}$, such that $d>\left|\varepsilon_{q}\right|$, the bulk state is gapped since the individual gaps overlap resulting in the global gap $\left(-d+\left|\varepsilon_{q}\right|, d-\left|\varepsilon_{q}\right|\right)$. We find that for the general normal-reflection BCs (3.5), describing a boundary that is an interface with vacuum (sample termination) or an insulator, there always exists a nondegenerate Majorana bound state at the Fermi level $\epsilon=0$ in this gapped bulk state.

mitigate it. In Sec. V, we derive our central result, the existence of the Majorana bound state. In Sec. VI, we generalize this analysis to the case of arbitrary number of Fermi surfaces. As examples of the microscopic realization of the general low-energy model for 1FS, in Sec. VII, we present the model of a quantum wire and, in Sec. VIII, the model of the edge

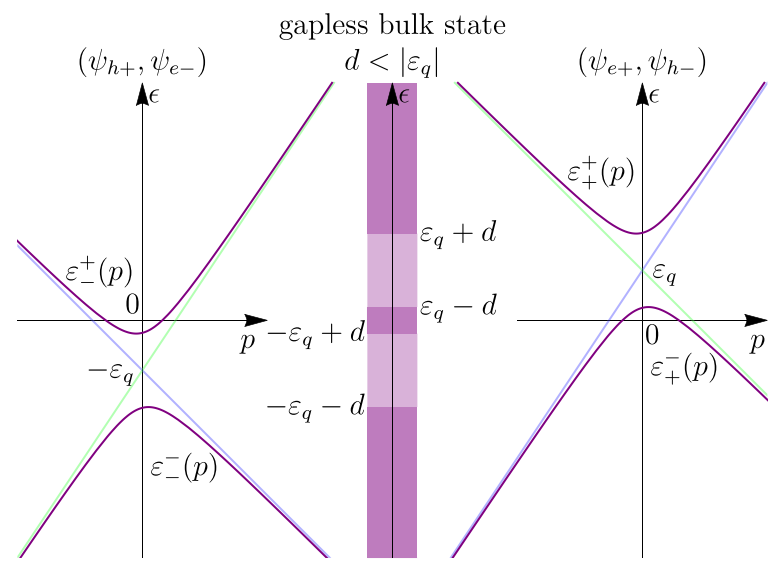

FIG. 5. Gapless bulk state despite the presence of the superconducting pairing field. In the presence of the Fermi-point mismatch, if the amplitude $\Delta_{0}$ of the pairing field $\Delta_{q}(x)=\Delta_{0} e^{i q x}$ [Eq. (4.1)] is small, such that $d<\left|\varepsilon_{q}\right|$ [Eqs. (4.5) and (4.7)], the individual energy gaps $\left(\varepsilon_{q}-d, \varepsilon_{q}+d\right)$ and $\left(-\varepsilon_{q}-d,-\varepsilon_{q}+d\right)$ in the bulk spectrum (4.6) around $\pm k_{0}$ points do not overlap, and hence, the bulk state is gapless. Bound states cannot exist in this regime. 
of a quantum spin Hall system in proximity of the magnetic material. In the concluding Sec. IX, we discuss the relation of our low-energy symmetry-based approach to studying bound states to the topological aspect of the system and provide an outlook. In the Appendix, we analyze the effect of TR symmetries $\mathcal{T}_{ \pm}$with $\mathcal{T}_{ \pm}^{2}= \pm 1$.

Before we proceed, we mention that recently, Ref. [45] came out, where a similar main conclusion about the existence of Majorana bound states was reached using a similar low-energy model. We discuss the main differences between our work and Ref. [45] in Sec. X after having presented our results.

\section{GENERAL LOW-ENERGY HAMILTONIAN FOR ONE FERMI SURFACE}

We start by deriving the most general form of the lowenergy BdG Hamiltonian for the case of 1FS.

For a real spinful electron system, creating 1FS requires some spin-orbit or magnetic effects, or their combination, in order to properly split the bands. An example of a 1FS system with spin-orbit interaction, but absent magnetic effects, is the edge of a 2D quantum spin Hall (QSH) system [2,46], Fig. 2 (anticipating our findings, magnetic effects that break TR symmetry $\mathcal{T}_{e-}$ are still required in such system in order to create a boundary). An example of a 1FS system with only magnetic but no spin-orbit effects is a quantum wire with a simple quadratic spectrum and Zeeman effect; inducing superconductivity in it at energies below the Zeeman splitting is only possible for a spin-triplet pairing field. In order to induce superconductivity from a spin-singlet pairing field, spin-orbit interactions are necessary, which amounts to the proposal of Refs. [3,4], Fig. 1.

Suppose $\mathcal{E}_{e}(k)$ is the normal-state electron band of the underlying microscopic model that crosses the Fermi level at two Fermi points assumed to be at momenta $\pm k_{ \pm} \gtrless 0$ in the 1D Brillouin zone, $\mathcal{E}_{e}\left( \pm k_{ \pm}\right)=0$; all energies will be measured relative to the Fermi level. Importantly, since we generally assume no symmetries, besides CC, the Fermi points are not necessarily at opposite momenta; if $k_{+} \neq k_{-}$, we will refer to such situation as the Fermi-point mismatch, Fig. 3. The Fermi-point mismatch could be prohibited by a symmetry (spatial, TR $\mathcal{T}_{e \pm}$, or their combination; see, e.g., the Appendix) that relates $k \rightarrow-k$ and hence imposes the constraint $\mathcal{E}_{e}(k)=\mathcal{E}_{e}(-k)$.

The corresponding hole spectrum in the normal state (in the absence of superconducting pairing) is $\mathcal{E}_{h}(k)=-\mathcal{E}_{e}(-k)$ (obtained by applying $\mathrm{CC}$ symmetry $\mathcal{C}_{+}$; see below). The crossings of the electron and hole normal-state spectra occur at two opposite momenta $\pm k_{0}$, where

$$
\mathcal{E}_{e}\left( \pm k_{0}\right)=-\mathcal{E}_{e}\left(\mp k_{0}\right) \text {. }
$$

For present Fermi-point mismatch, $k_{+} \neq k_{-}$, the crossings occur at finite energies $\pm \varepsilon_{0}$, respectively.

As we show below, in order for the gapped superconducting state to be induced in such system, the superconducting pairing field has to overcome the effect of the Fermi-point mismatch. Therefore, considering the low-energy model, we assume the mismatch small compared to the nonlinearity scale of the band $\mathcal{E}_{e}(k)$, i.e., that, at the very least, $\left|k_{+}-k_{-}\right| \ll k_{ \pm}$.
Under this approximation, the energy and momentum of the crossing points can be found as

$$
\varepsilon_{0}=-\frac{v_{+} v_{-}}{v_{+}+v_{-}}\left(k_{+}-k_{-}\right), \quad k_{0}=\frac{v_{+} k_{+}+v_{-} k_{-}}{v_{+}+v_{-}},
$$

from the linearized electron spectrum

$$
\mathcal{E}_{e}(k) \approx \pm v_{ \pm}\left(k \mp k_{ \pm}\right)= \pm v_{ \pm}\left(k \mp k_{0}\right) \pm \varepsilon_{0}
$$

around the Fermi or crossing points. To leading order, the velocities

$$
v_{ \pm}= \pm \partial_{k} \mathcal{E}_{e}\left( \pm k_{ \pm}\right) \approx \pm \partial_{k} \mathcal{E}_{e}\left( \pm k_{0}\right)>0
$$

at the Fermi points $\pm k_{ \pm}$and at the crossing points $\pm k_{0}$ are equal.

This expansion of the underlying spectrum $\mathcal{E}_{e}(k)$ about the crossing points $\pm k_{0}$ to the linear order gives the low-energy electron Hamiltonian

$$
\hat{H}_{e}(\hat{p})=\left(\begin{array}{cc}
v_{+} \hat{p}+\varepsilon_{0} & 0 \\
0 & -v_{-} \hat{p}-\varepsilon_{0}
\end{array}\right)
$$

for the low-energy electron wave function

$$
\hat{\psi}_{e}(x)=\left(\begin{array}{l}
\psi_{e+}(x) \\
\psi_{e-}(x)
\end{array}\right) .
$$

Here, $\hat{p}=-i \partial_{x}$ is the momentum operator corresponding to the momentum deviations from the crossing points $\pm k_{0}$.

In the BdG formalism of superconductivity [30], one introduces a complementary hole wave function

$$
\hat{\psi}_{h}(x)=\left(\begin{array}{l}
\psi_{h+}(x) \\
\psi_{h-}(x)
\end{array}\right),
$$

so that the full BdG wave function reads

$$
\hat{\psi}(x)=\left(\begin{array}{c}
\hat{\psi}_{e}(x) \\
\hat{\psi}_{h}(x)
\end{array}\right)=\left(\begin{array}{c}
\psi_{e+}(x) \\
\psi_{e-}(x) \\
\psi_{h+}(x) \\
\psi_{h-}(x)
\end{array}\right) .
$$

The structure of the $\mathrm{BdG}$ Hamiltonian $\hat{H}(\hat{p})$ in this electron-hole space is governed by charge-conjugation (CC) symmetry. A Hamiltonian $\hat{H}(\hat{p})$ satisfies $\mathrm{CC}$ symmetry if there exists an antiunitary $\mathrm{CC}$ operation

$$
\mathcal{C}_{+}=\hat{C}_{+} \mathcal{K},
$$

where $\hat{C}_{+}$is a unitary matrix and $\mathcal{K}$ is the complexconjugation operation, under which the Hamiltonian changes its sign,

$$
\hat{C}_{ \pm}[\hat{H}(\hat{p})]^{*} \hat{C}_{ \pm}^{\dagger}=-\hat{H}(\hat{p}),
$$

with $\hat{p}^{*}=-\hat{p}$. A constraint is also imposed that the CC operation squares to either \pm 1 . For a spinful electron system with broken spin symmetry, the physical CC operation $\mathcal{C}_{+}$ is the one that squares to +1 (which we emphasize with the subscript in $\mathcal{C}_{+}$), i.e.,

$$
\mathcal{C}_{+}^{2}=\hat{C}_{+} \hat{C}_{+}^{*}=\hat{1}_{4} ;
$$

it is the one that ensures the antisymmetry of the pairing field, in accord with the Fermi statistics. This symmetry alone 
realizes systems of class D of the topological classification scheme [31].

In the basis of Eq. (2.5), without loss of generality, the CC operation can always be brought to the form

$$
\hat{C}_{+}=\tau_{x} \otimes \hat{1}_{2}
$$

by the appropriate choice of electron and holes basis states, where $\tau_{x}$ is the second Pauli matrix and $\hat{1}_{2}$ is a $2 \times 2$ unit matrix. So, the action of the $\mathrm{CC}$ operation on the wave function reads

$$
\mathcal{C}_{+} \hat{\psi}(x)=\left(\begin{array}{c}
\hat{\psi}_{h}^{*}(x) \\
\hat{\psi}_{e}^{*}(x)
\end{array}\right)
$$

Note that the CC operation does not alter the coordinate, which will be important for the analysis of the CC symmetry of the $\mathrm{BCs}$.

Once the $\mathrm{CC}$ operation $\mathcal{C}_{+}$is specified, the most general form of the $\mathrm{BdG}$ Hamiltonian allowed by $\mathcal{C}_{+}$symmetry reads

$$
\hat{H}(\hat{p})=\left(\begin{array}{cc}
\hat{H}_{e}(\hat{p}) & \hat{\Delta}(x) \\
\hat{\Delta}^{\dagger}(x) & -\hat{H}_{e}^{\mathrm{T}}(-\hat{p})
\end{array}\right),
$$

in the basis (2.5). The form $-\hat{H}_{e}^{\mathrm{T}}(-\hat{p})$ of the hole Hamiltonian is fixed by that of the electron one [Eq. (2.2)] and the superconducting pairing field matrix $\hat{\Delta}(x)$ must be antisymmetric, $\hat{\Delta}^{\mathrm{T}}(x)=-\hat{\Delta}(x)$. Here, $\mathrm{T}$ denotes matrix transposition. And since in the $1 \mathrm{FS}$ case it is a $2 \times 2$ matrix, its form is uniquely fixed by this, $\hat{\Delta}(x)=i \tau_{y} \Delta(x)$. The pairing field of the lowenergy model (2.10) is therefore fully described by one complex function $\Delta(x)$. This holds regardless of the actual underlying spin structure of the pairing field (this can mean, however, that pairing fields with some spin structures cannot be induced; see discussion in Sec. VII C for the generalized quantum-wire model).

So, for $1 \mathrm{FS}$, the most general form of the $\mathcal{C}_{+}$-symmetric BdG low-energy Hamiltonian reads

$$
\hat{H}(\hat{p})=\left(\begin{array}{cccc}
v_{+} \hat{p}+\varepsilon_{0} & 0 & 0 & \Delta(x) \\
0 & -v_{-} \hat{p}-\varepsilon_{0} & -\Delta(x) & 0 \\
0 & -\Delta^{*}(x) & v_{+} \hat{p}-\varepsilon_{0} & 0 \\
\Delta^{*}(x) & 0 & 0 & -v_{-} \hat{p}+\varepsilon_{0}
\end{array}\right) .
$$

The pairs $\left(\psi_{e+}(x), \psi_{h-}(x)\right)$ and $\left(\psi_{h+}(x), \psi_{e-}(x)\right)$ of the wave-function (2.5) components of the states around $\pm k_{0}$ points, respectively, are not coupled by the bulk Hamiltonian (2.11).

The normal-state spectrum in the absence of the pairing field $\Delta(x)$ for the full BdG Hamiltonian (2.11), including the hole states, is shown in Fig. 3. Note that both the electron $\psi_{e \pm}(x)$ and hole $\psi_{h \pm}(x)$ components of the BdG wave function (2.5) are labeled with \pm according to their propagation direction (right and left, respectively, in the geometry of Fig. 3) as set by the signs $\pm v_{ \pm} \gtrless 0$ of their velocities. The electron components $\psi_{e \pm}(x)$ correspond to $\pm k_{0}$ momenta, respectively, by construction, while the hole components $\psi_{h \pm}(x)$ correspond to $\mp k_{0}$.

\section{GENERAL CHARGE-CONJUGATION-SYMMETRIC BOUNDARY CONDITIONS}

We now derive the most general form of the $\mathrm{BCs}$ for the BdG Hamiltonian (2.11) for 1FS, restricted only by the probability-current conservation principle and $\mathrm{CC}$ symmetry $\mathcal{C}_{+}$.

We assume that the low-energy system is half-infinite and occupies the region $x \geqslant 0 ; x=0$ is its boundary. The lowenergy wave function $\hat{\psi}(x)$ [Eq. (2.5)] is not defined in the region $x<0$; physically, this means that there is a large excitation gap in the region $x<0$ in the underlying microscopic model, which renders it inaccessible for the low-energy excitations of $\hat{\psi}(x)$.

The most general possible form of the BCs of a continuum model is governed [33-43] by the fundamental principle of the conservation of the probability current $j(x)$, which follows from the Hermiticity of the Hamiltonian, which, in turn, follows from the norm conservation of the wave function. For a half-infinite system, this principle takes the form of the current nullification at the boundary,

$$
j(0)=0 .
$$

For an arbitrary 1D continuum model, such BCs have been derived in Ref. [40]. For the linear-in-momentum Hamiltonian (2.11), the probability current

$$
\begin{gathered}
j(x)=j_{e}(x)+j_{h}(x), \quad j_{v}(x)=j_{v+}(x)+j_{v-}(x), \\
j_{v \pm}(x)= \pm v_{ \pm} \psi_{v \pm}^{*}(x) \psi_{\nu \pm}(x), \quad v=e, h,
\end{gathered}
$$

is a diagonal quadratic form of the wave-function components and the most general form of the BCs nullifying it reads

$$
\hat{\bar{\psi}}_{+}(0)=\hat{U} \hat{\bar{\psi}}_{-}(0) \text {. }
$$

Here,

$$
\hat{\bar{\psi}}_{ \pm}(x)=\left(\begin{array}{c}
\sqrt{v_{ \pm}} \psi_{e \pm}(x) \\
\sqrt{v_{ \pm}} \psi_{h \pm}(x)
\end{array}\right)
$$

are the vectors that group the wave-function components (2.5) with the same sign \pm of velocities (right- and left-moving states, respectively) and

$$
\hat{U}=\left(\begin{array}{ll}
u_{e e} & u_{e h} \\
u_{h e} & u_{h h}
\end{array}\right), \quad \hat{U} \hat{U}^{\dagger}=\hat{1},
$$

is a $\mathrm{U}(2)$ unitary matrix. Thus, all possible BCs form a family parametrized by the unitary matrix $\hat{U}$. Each instance of $\hat{U}$ delivers one possible set of BCs. The structure of the BCs (3.4) is particularly transparent and has a natural physical interpretation as a scattering process off the boundary between the incident (left-moving) $\hat{\bar{\psi}}_{-}(x)$ and reflected (right-moving) $\hat{\bar{\psi}}_{+}(x)$ waves, where $\hat{U}$ plays the role of the scattering matrix.

For arbitrary $\hat{U}$, these $\mathrm{BCs}$ generally break $\mathcal{C}_{+}$symmetry, in which case the system with a boundary does not have $\mathcal{C}_{+}$symmetry even though the bulk Hamiltonian (2.11) does. However, in order for the bound states to represent the topological properties of the bulk stemming from a certain symmetry, the system with a boundary must satisfy that symmetry. Within the continuum-model formalism, this means that the BCs must also satisfy that symmetry [41]. In turn, 
the latter means that the wave function transformed under that symmetry operation also satisfies the BCs (BCs essentially restrict the Hilbert space, and since the transformed wave function must also belong to the same Hilbert space, it must satisfy those BCs). This introduces constraints on the allowed form of $\hat{U}$. For the CC symmetry $\mathcal{C}_{+}$in question, inserting the transformed wave function $\mathcal{C}_{+} \hat{\psi}(0)$ [Eq. (2.9)] into the BCs (3.4), we find that the transformed wave function satisfies them if the following constraints on $\hat{U}$ are satisfied:

$$
u_{h h}=u_{e e}^{*}, \quad u_{h e}=u_{e h}^{*} .
$$

(Note that, importantly, the $\mathrm{CC}$ operation $\mathcal{C}_{+}$does not alter the coordinate $x$ and thus leaves the geometry of system intact; hence, the system with a boundary can, in principle, be $\mathcal{C}_{+}$-symmetric.) From this, choosing $u_{e e}$ and $u_{e h}$ as the independent matrix elements and combining with the unitarity of $\hat{U}$, we obtain the following constraints:

$$
u_{e e} u_{e e}^{*}+u_{e h} u_{e h}^{*}=1, \quad u_{e e} u_{e h}=0 .
$$

Thus, there are only two options. The first option is when $u_{e h}=0$, and

$$
\hat{U}=\left(\begin{array}{cc}
U_{e e} & 0 \\
0 & U_{e e}^{*}
\end{array}\right), \quad\left|U_{e e}\right|=1 ;
$$

the spelled out Eq. (3.4) takes the form

$$
\begin{aligned}
& \sqrt{v_{+}} \psi_{e+}(0)=U_{e e} \sqrt{v_{-}} \psi_{e-}(0), \\
& \sqrt{v_{+}} \psi_{h+}(0)=U_{e e}^{*} \sqrt{v_{-}} \psi_{h-}(0) .
\end{aligned}
$$

The second option is when $u_{e e}=0$, and

$$
\hat{U}=\left(\begin{array}{cc}
0 & U_{e h} \\
U_{e h}^{*} & 0
\end{array}\right), \quad\left|U_{e h}\right|=1 ;
$$

the spelled out Eq. (3.4) takes the form

$$
\begin{aligned}
& \sqrt{v_{+}} \psi_{e+}(0)=U_{e h} \sqrt{v_{-}} \psi_{h-}(0), \\
& \sqrt{v_{+}} \psi_{h+}(0)=U_{e h}^{*} \sqrt{v_{-}} \psi_{e-}(0) .
\end{aligned}
$$

Thus, we obtain that the most general BCs subject only to the current nullification principle and $\mathrm{CC}$ symmetry $\mathcal{C}_{+}$consist of two families, Eqs. (3.5) and (3.6), each parametrized by the respective scattering phase factors $U_{e e}$ and $U_{e h}$; each value of $U_{e e}$ or $U_{e h}$ corresponds to one possible set of BCs of the respective family. Note that the two families are disconnected in the parameter space of the $\hat{U} \in \mathrm{U}(2)$ matrix manifold and can never be connected without breaking $\mathcal{C}_{+}$symmetry. Also note that, for each family, one of the equations in the BCs can be obtained from the other by the $\mathcal{C}_{+}$operation, underscoring their $\mathcal{C}_{+}$symmetry.

In the BCs (3.5), there is no scattering between the electron and hole components of the wave function; the electron $j_{e}(0)=j_{e+}(0)+j_{e-}(0)=0$ and hole $j_{h}(0)=j_{h+}(0)+$ $j_{h-}(0)=0$ contributions to the total current (3.2) vanish individually. On the other hand, in the BCs (3.6), there is scattering only between the electron and hole components; the combinations $j_{e+}(0)+j_{h-}(0)=0$ and $j_{e-}(0)+j_{h+}(0)=0$, each involving parts of the electron and hole currents, vanish individually. Accordingly, we term these BCs the normalreflection [Eq. (3.5)] and Andreev-reflection [Eq. (3.6)] BCs, respectively. The BCs are illustrated schematically in Fig. 6.

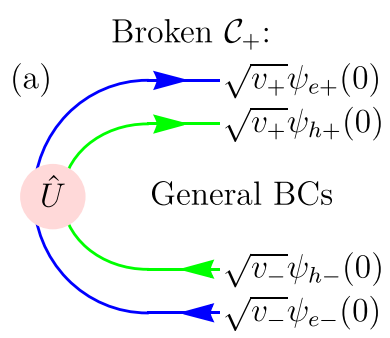

Preserved $\mathcal{C}_{+}$:
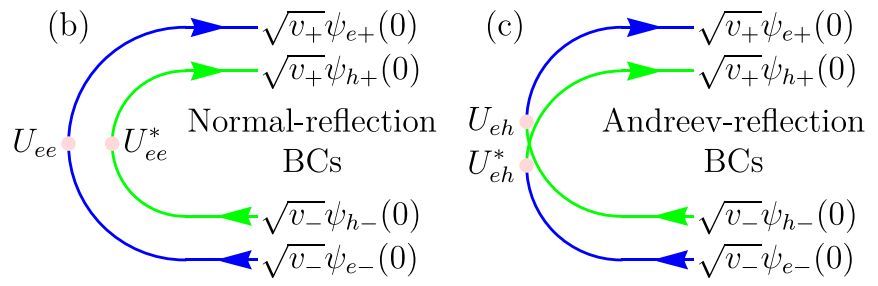

FIG. 6. Illustration of the boundary conditions (BCs) for the low-energy model of a 1D superconductor with one Fermi surface (1FS) with the Hamiltonian $\hat{H}(\hat{p})$ [Eq. (2.11)]. (a) The most general form (3.4) of the BCs subject only to the fundamental principle of probability-current conservation [Eqs. (3.1) and (3.2)] is parametrized by the unitary matrix $\hat{U} \in \mathrm{U}(2)$, which has a natural interpretation of the scattering matrix between the incident (left-moving) $\left(\psi_{e-}, \psi_{h-}\right)$ and reflected (right-moving) $\left(\psi_{e+}, \psi_{h+}\right)$ components of the BdG wave function (2.5), Fig. 3. Under chargeconjugation (CC) symmetry $\mathcal{C}_{+}$[Eqs. (2.6), (2.8), and (2.9)], there are only two allowed disconnected subfamilies of these BCs, which we term (b) normal-reflection [Eq. (3.5)] and (c) Andreev-reflection [Eq. (3.6)] BCs. (b) In the normal-reflection BCs, electrons are reflected as electrons and holes as holes. The boundary of a superconductor with an insulator, of interest in this work, can only be described by normal-reflection BCs. (c) In the Andreev-reflection BCs, electrons are completely reflected as holes and vice versa. Such $\mathrm{BCs}$ cannot be defined in the normal state and cannot represent such boundary.

The main crucial difference between the two families of $\mathrm{BCs}$ is that the normal-reflection BCs (3.5) are well-defined already in the normal state, i.e., in the absence of the superconducting pairing field $\Delta(x)$ and just for the electron part $\hat{\psi}_{e}(x)$ of the BdG wave function $\hat{\psi}(x)$ [Eq. (2.5)], without introducing the hole part $\hat{\psi}_{h}(x)$. Indeed, the first BC in Eq. (3.5) involves only the components of $\hat{\psi}_{e}(x)$ and is the most general form of the BC [33,37-42] for the electron Hamiltonian $\hat{H}_{e}(\hat{p})\left[\right.$ Eq. (2.2)] with the probability current $j_{e}(x)$ [Eq. (3.2)] subject only to the current nullification principle $j_{e}(0)=0$.

In contrast, the Andreev-reflection BCs (3.6) cannot be defined in the normal state: they can be defined only in the presence of the hole part $\hat{\psi}_{h}(x)$, which implies the presence of superconductivity at the boundary in some form even in the absence of the pairing field $\Delta(x)$. For example, one plausible physical realization of the Andreev-reflection BCs could be that the inaccessible region $x<0$ is a superconductor with a much larger gap.

In this work, our focus is on the bound states of a physical quasi-1D sample that is terminated (as in Fig. 1) or interfaced with an insulator (as in Fig. 2), when the inaccessible region $x<0$ is a vacuum or an insulating material. According 
to the above considerations, the boundary of such system with a well-defined normal state can be represented in the low-energy model only by the normal-reflection BCs (3.5). Therefore, in the remainder of the paper, we will consider only the normal-reflection BCs (3.5), while the Andreev-reflection BCs (3.6) will be explored elsewhere.

\section{FERMI-POINT MISMATCH, COORDINATE-DEPENDENT PAIRING FIELD, BULK SPECTRUM}

The derived low-energy Hamiltonian (2.11) is valid for an arbitrary coordinate dependence of the pairing field $\Delta(x)$. Which dependence is actually favored in a real system may depend on various factors and be nonobvious. As the main application, we have in mind the setups where superconductivity is induced in the quasi-1D system due to the proximity effect of a nearby superconductor, as in Figs. 1 and 2. Strictly speaking, within the mean-field approach to the interacting many-body Hamiltonian, the favored form of the pairing field must be found by minimizing the energy of the BCS Slaterdeterminant trial many-body state of this coupled system. Without the Fermi-point mismatch, it is likely that the induced pairing field is coordinate-independent, $\Delta(x)=\Delta_{0}$. With the Fermi-point mismatch, however, a plausible scenario is that the induced pairing field could have a periodic behavior associated with the momentum mismatch $k_{+}-k_{-} \neq 0$, akin to the Larkin-Ovchinnikov-Fulde-Ferrel state [50,51].

Let us examine a one-harmonic periodic coordinate dependence

$$
\Delta_{q}(x)=\Delta_{0} e^{i q x}, \quad \Delta_{0}=\left|\Delta_{0}\right| e^{-i \delta},
$$

characterized by some momentum $q$, which we discuss below. This explicit coordinate dependence can be eliminated from the Hamiltonian $\left.\hat{H}(\hat{p})\right|_{\Delta(x)=\Delta_{q}(x) ; \varepsilon_{0}}$ [Eq. (2.11)] with the pairing field $\Delta_{q}(x)$ and Fermi-point-mismatch energy $\varepsilon_{0}$ by the following transformation of the wave function:

$$
\hat{\psi}(x)=\left(\begin{array}{c}
e^{i\left(+1-\bar{v}_{0}\right) \frac{q}{2} x} \psi_{e+}^{\prime}(x) \\
e^{i\left(+1+\bar{v}_{0}\right) \frac{q}{2} x} \psi_{e-}^{\prime}(x) \\
e^{i\left(-1+\bar{v}_{0}\right) \frac{q}{2} x} \psi_{h+}^{\prime}(x) \\
e^{i\left(-1-\bar{v}_{0}\right) \frac{q}{2} x} \psi_{h-}^{\prime}(x)
\end{array}\right), \quad \hat{\psi}^{\prime}(x)=\left(\begin{array}{c}
\psi_{e+}^{\prime}(x) \\
\psi_{e-}^{\prime}(x) \\
\psi_{h+}^{\prime}(x) \\
\psi_{h-}^{\prime}(x)
\end{array}\right)
$$

where

$$
\bar{v}_{0}=\frac{v_{0}}{v_{z}}, \quad v_{0, z}=\frac{1}{2}\left(v_{+} \mp v_{-}\right) .
$$

It essentially amounts to introducing proper individual momentum shifts for the wave-function components, which can be deduced by analyzing the Hamiltonian in momentum space. This way, we find that the Hamiltonian for the wave function $\hat{\psi}^{\prime}(x)$ has the form

$$
\left.\hat{H}^{\prime}(\hat{p}) \equiv \hat{H}(\hat{p})\right|_{\Delta(x)=\Delta_{0} ; \varepsilon_{0} \rightarrow \varepsilon_{q}}
$$

of Eq. (2.11), but with the coordinate-independent pairing field $\Delta_{0}$ and modified, $q$-dependent mismatch energy [Eq. (2.1)]

$$
\varepsilon_{q}=\varepsilon_{0}+\frac{v_{+} v_{-}}{v_{+}+v_{-}} q=\frac{v_{+} v_{-}}{v_{+}+v_{-}}\left[q-\left(k_{+}-k_{-}\right)\right] .
$$

The Hamiltonian $\hat{H}^{\prime}(\hat{p})$ is thus effectively translationally invariant and its bulk eigenstates for an infinite system can be characterized by the momentum quantum number $p$ [although different components of the original wave function $\hat{\psi}(x)$ have different momenta, as per Eq. (4.2)]. Hence, the bulk spectrum of both Hamiltonians $\hat{H}^{\prime}(\hat{p})$ and $\left.\hat{H}(\hat{p})\right|_{\Delta(x)=\Delta_{q}(x) ; \varepsilon_{0}}$ consists of the bands

$$
\begin{gathered}
\varepsilon_{+}^{ \pm}(p)=+\varepsilon_{q}+v_{0} p \pm \sqrt{v_{z}^{2} p^{2}+\left|\Delta_{0}\right|^{2}} \\
\varepsilon_{-}^{ \pm}(p)=-\varepsilon_{q}+v_{0} p \pm \sqrt{v_{z}^{2} p^{2}+\left|\Delta_{0}\right|^{2}}
\end{gathered}
$$

for the decoupled pairs $\left(\psi_{e+}(x), \psi_{h-}(x)\right)$ and $\left(\psi_{h+}(x), \psi_{e-}(x)\right)$ of components around $\pm k_{0}$ momenta, respectively, as plotted in Figs. 4 and 5. We observe that the individual energy gaps $\left(\varepsilon_{q}-d, \varepsilon_{q}+d\right)$ and $\left(-\varepsilon_{q}-d,-\varepsilon_{q}+d\right)$ with

$$
d=\left|\Delta_{0}\right| \sqrt{1-\bar{v}_{0}^{2}}
$$

open up in the bulk spectrum (4.6) around $\pm k_{0}$ momenta, respectively, for any value of the pairing field $\Delta_{0}$. However, when $d<\left|\varepsilon_{q}\right|$, these individual gaps do not overlap and the bulk state remains gapless despite the presence of the pairing field (Fig. 5). Only when $d>\left|\varepsilon_{q}\right|$ exceeds the modified mismatch energy, the individual gaps overlap, resulting in the global energy gap $\left(-d+\left|\varepsilon_{q}\right|, d-\left|\varepsilon_{q}\right|\right)$ centered around the Fermi level $\epsilon=0$ (Fig. 4). Thus, whenever the modified mismatch energy $\varepsilon_{q} \neq 0$ is nonzero, there is a threshold for creating a gapped bulk superconducting state $[20,44]$.

The modified mismatch energy $\varepsilon_{q}=0$ is absent when $q=k_{+}-k_{-}$. Only in this case there is no threshold for gap opening, which would suggest that such $q$ in Eq. (4.1) should be favorable. However, this means that in the nearby superconductor (in setups such as in Fig. 1), which is the source of superconductivity, the pairing field must have a similar coordinate dependence at least in some region close to the quasi-1D system, which would cause a penalty in the gradient energy. Therefore, which value of $q$ (whether 0 or $k_{+}-k_{-}$, or some intermediate value) minimizes the ground-state energy of the interacting many-body system cannot be answered without carrying out the minimization procedure for the whole coupled system. This question is beyond the focus of this work and we do not attempt to answer it here. Instead, we calculate the bound states for any Fermi-point mismatch $k_{+}-k_{-}$and for the coordinate dependence (4.1) of the pairing field with any $q$ and demonstrate that a Majorana bound state does exist regardless of their values, as long as the superconducting state is gapped (Fig. 4).

\section{EVER-PRESENT MAJORANA BOUND STATE FOR ONE FERMI SURFACE}

Having derived the general forms of the bulk Hamiltonian [Eq. (2.11)] and normal-reflection BCs [Eq. (3.5)] for the case of 1FS, we now analytically calculate the bound states for the half-infinite system $x \geqslant 0$ with the pairing field of the form $\Delta_{q}(x)$ [Eq. (4.1)]. 
The calculation is straightforward. We first construct a general solution to the Schrödinger equation

$$
\hat{H}^{\prime}(\hat{p}) \hat{\psi}^{\prime}(x)=\epsilon \hat{\psi}^{\prime}(x)
$$

that decays into the bulk, as $x \rightarrow+\infty$. A decaying solution can exist only in the gapped superconducting state (Fig. 4), i.e., for $d>\left|\varepsilon_{q}\right|$ [Eqs. (4.5) and (4.7)] in the presence of the Fermi-point mismatch, at energies $\epsilon \in\left(-d+\left|\varepsilon_{q}\right|, d-\left|\varepsilon_{q}\right|\right)$ within the gap of the bulk spectrum (4.6). The general decaying solution is a linear combination of particular decaying solutions with complex momenta $p(\epsilon)$, obtained from the characteristic equation

$$
\operatorname{det}\left[\hat{H}^{\prime}(p)-\epsilon \hat{1}_{4}\right]=0 .
$$

There are two such particular solutions $\hat{\mathcal{X}}_{ \pm}(\epsilon) e^{i p_{ \pm}(\epsilon) x}$, with the momenta

$$
p_{ \pm}(\epsilon)=\frac{d}{v_{z}^{2}-v_{0}^{2}}\left(-\bar{v}_{0} \bar{\epsilon}_{ \pm}+i \sqrt{1-\bar{\epsilon}_{ \pm}^{2}}\right)
$$

and vectors

$$
\hat{\mathcal{X}}_{+}(\epsilon)=\left(\begin{array}{c}
\frac{1}{\sqrt{v_{+}}} X_{e+}(\epsilon) \\
0 \\
0 \\
\frac{1}{\sqrt{v_{-}}}
\end{array}\right), \quad \hat{\mathcal{X}}_{-}(\epsilon)=\left(\begin{array}{c}
0 \\
\frac{1}{\sqrt{v_{-}}} \\
\frac{1}{\sqrt{v_{+}}} X_{h+}(\epsilon) \\
0
\end{array}\right)
$$

where we denote

$$
\begin{aligned}
& X_{e+}(\epsilon)=e^{i\left[\Phi_{+}(\epsilon)-\delta\right]}, \quad X_{h+}(\epsilon)=-e^{i\left[\Phi_{-}(\epsilon)+\delta\right]}, \\
& e^{i \Phi_{ \pm}(\epsilon)}=\bar{\epsilon}_{ \pm}+i \sqrt{1-\bar{\epsilon}_{ \pm}^{2}}, \quad \bar{\epsilon}_{ \pm}=\frac{\epsilon \mp \varepsilon_{q}}{d} .
\end{aligned}
$$

We observe that the relation

$$
X_{h+}(\epsilon)=X_{e+}^{*}(-\epsilon)
$$

holds, which ultimately is a consequence of the CC symmetry $\mathcal{C}_{+}$and is key to the conclusion about the Majorana bound state at $\epsilon=0$.

These particular solutions originate from the pairs $\left(\psi_{e+}^{\prime}(x), \psi_{h_{-}}^{\prime}(x)\right)$ and $\left(\psi_{h_{+}}^{\prime}(x), \psi_{e-}^{\prime}(x)\right)$ of components around $\pm k_{0}$ momenta, respectively, which are decoupled in the bulk Hamiltonian. The general decaying solution reads

$$
\hat{\psi}^{\prime}(x)=c_{+} \hat{\mathcal{X}}_{+}(\epsilon) e^{i p_{+}(\epsilon) x}+c_{-} \hat{\mathcal{X}}_{-}(\epsilon) e^{i p_{-}(\epsilon) x},
$$

where $c_{ \pm}$are the free coefficients.

Inserting this wave function (5.5) via Eq. (4.2) into the normal-reflection BCs (3.5), we obtain a linear homogeneous system

$$
X_{e+}(\epsilon) c_{+}=U_{e e} c_{-}, \quad X_{h+}(\epsilon) c_{-}=U_{e e}^{*} c_{+}
$$

of equations for the unknown coefficients $c_{ \pm}$, in which the energy $\epsilon$ is a parameter. This system has nontrivial solutions, which are the sought bound states, if its determinant is zero, i.e., when

$$
X_{e+}(\epsilon) X_{h+}(\epsilon)=U_{e e} U_{e e}^{*} .
$$

The scattering phase factor $U_{e e}$ drops out due to $U_{e e} U_{e e}^{*}=1$; so does the phase $\delta$ of the pairing field (4.1) contained in Eq. (5.3). Using the key relation (5.4), the equation for the energy $\epsilon$ of possible bound states becomes

$$
X_{e+}(\epsilon) X_{e+}^{*}(-\epsilon)=1 \text {. }
$$

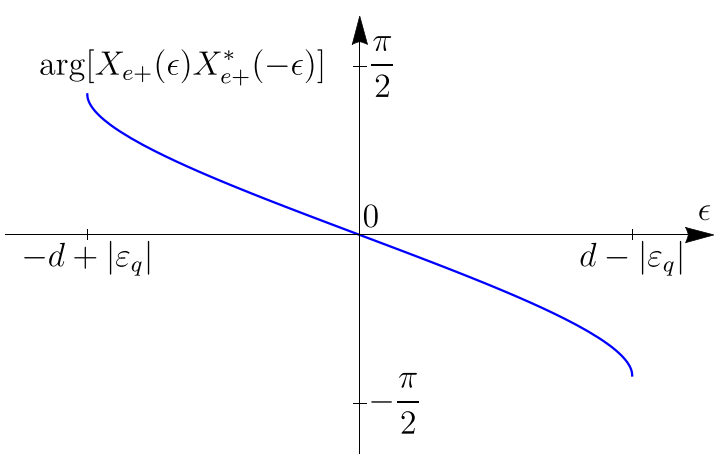

FIG. 7. The argument of the left-hand side $X_{e+}(\epsilon) X_{e+}^{*}(-\epsilon)$ [Eq. (5.3)] of Eq. (5.6) determining the energies of possible bound states, as a function of energy $\epsilon \in\left(-d+\left|\varepsilon_{q}\right|, d-\left|\varepsilon_{q}\right|\right)$ within the gap. This plot explicitly shows that there exists a Majorana bound state at $\epsilon=0$ and there are no other bound-state solutions.

In this form, it is evident that $\epsilon=0$ is a solution, which describes a Majorana bound state. In Fig. 7, we plot the argument of the left-hand side of Eq. (5.6), which also shows that there are no other bound-state solutions.

Hence, we arrive at the central result of this work, that, as long as a gapped superconducting state can be induced in a quasi-1D system with 1FS, there always exists a Majorana bound state at its boundary with an insulator (where the latter could be an actual insulator material or vacuum, for a terminated sample), as illustrated in Fig. 4. As already explained in Sec. I, since this claim has been proven for the low-energy model with the most general forms of the bulk Hamiltonian and normal-reflection BCs, it holds for the whole class of systems: any microscopic model with such general properties will reduce to an instance of the derived general low-energy model in the low-energy limit and will thus host Majorana bound states regardless of its other details. Although the presented proof is self-contained, it is nonetheless insightful to illustrate this latter point for specific microscopic models, which we do in Secs. VII and VIII.

We stress the $\epsilon=0$ Majorana bound state exists specifically for normal-reflection BCs (3.5), and only this family of general $\mathcal{C}_{+}$-symmetric BCs can represent the boundary with an insulator, which is the focus of the present work. As explained in Sec. III, Andreev-reflection BCs cannot represent such boundary, since they imply the presence of superconductivity in some form even for absent pairing field $\Delta(x)$. The analysis of Andreev-reflection BCs is for this reason beyond the focus of this work.

\section{EVER-PRESENT MAJORANA BOUND STATE FOR ODD NUMBER OF FERMI SURFACES}

In this section, we generalize the above-presented formalism and result to the case of arbitrary number $N \geqslant 1$ of FSs. The wave function for the low-energy BdG model now reads

$$
\hat{\psi}(x)=\left(\begin{array}{c}
\hat{\psi}_{e+}(x) \\
\hat{\psi}_{e-}(x) \\
\hat{\psi}_{h+}(x) \\
\hat{\psi}_{h-}(x)
\end{array}\right)
$$


where

$$
\hat{\psi}_{\nu \pm}(x)=\left(\begin{array}{c}
\psi_{v \pm}^{1}(x) \\
\cdots \\
\psi_{v \pm}^{N}(x)
\end{array}\right), \quad v=e, h,
$$

are now vectors in the $N$-dimensional FS space. The matrix of the CC symmetry operation now reads

$$
\hat{C}_{+}=\tau_{x} \otimes \hat{1}_{2} \otimes \hat{1}_{N} .
$$

We will consider the case when the separations between the FSs are much larger than the low-energy scales, set by the superconducting pairing field and the Fermi-point mismatch of each FS. In this case, potential superconducting pairing between different FSs is energetically unfavorable and we neglect it. Accordingly, the general Hamiltonian

$$
\hat{H}(\hat{p})=\left(\begin{array}{cccc}
\hat{v}_{+} \hat{p}+\hat{\varepsilon}_{0} & \hat{0} & \hat{0} & \hat{\Delta}(x) \\
\hat{0} & -\hat{v}_{-} \hat{p}-\hat{\varepsilon}_{0} & -\hat{\Delta}(x) & \hat{0} \\
\hat{0} & -\hat{\Delta}^{\dagger}(x) & \hat{v}_{+} \hat{p}-\hat{\varepsilon}_{0} & \hat{0} \\
\hat{\Delta}^{\dagger}(x) & \hat{0} & \hat{0} & -\hat{v}_{-} \hat{p}+\hat{\varepsilon}_{0}
\end{array}\right)
$$

is diagonal in the FS space and has the same structure in the Gor'kov-Nambu space as the Hamiltonian (2.11) for 1FS. The velocities

$$
\hat{v}_{ \pm}=\operatorname{diag}\left(v_{ \pm}^{1}, \ldots, v_{ \pm}^{N}\right), \quad v_{ \pm}^{n}>0,
$$

energy shift

$$
\hat{\varepsilon}_{0}=\operatorname{diag}\left(\varepsilon_{0}^{1}, \ldots, \varepsilon_{0}^{N}\right),
$$

and pairing field

$$
\hat{\Delta}(x)=\operatorname{diag}\left(\Delta^{1}(x), \ldots, \Delta^{N}(x)\right)
$$

are now diagonal matrices in the FS space.

Turning to the $\mathrm{BCs}$, the expressions for the probability current (3.2) now read

$$
j_{v \pm}(x)= \pm \hat{\psi}_{v \pm}^{\dagger}(x) \hat{v}_{ \pm} \hat{\psi}_{v \pm}(x)= \pm \hat{\bar{\psi}}_{v \pm}^{\dagger}(x) \hat{\bar{\psi}}_{v \pm}(x)
$$

where

$$
\hat{\bar{\psi}}_{ \pm}(x)=\left(\begin{array}{c}
\hat{\bar{\psi}}_{e \pm}(x) \\
\hat{\bar{\psi}}_{h \pm}(x)
\end{array}\right), \quad \hat{\bar{\psi}}_{v \pm}(x)=\left(\begin{array}{c}
\sqrt{v_{ \pm}^{1}} \psi_{v \pm}^{1}(x) \\
\ldots \\
\sqrt{v_{ \pm}^{N}} \psi_{v \pm}^{N}(x)
\end{array}\right) .
$$

According to Ref. [40], the BCs of the most general form, satisfying only the current-nullification principle, have the form

$$
\hat{\bar{\psi}}_{+}(0)=\hat{U} \hat{\bar{\psi}}_{-}(0)
$$

with $\hat{\bar{\psi}}_{ \pm}(x)$ from Eq. (6.3) and an arbitrary unitary matrix

$$
\hat{U}=\left(\begin{array}{ll}
\hat{u}_{e e} & \hat{u}_{e h} \\
\hat{u}_{h e} & \hat{u}_{h h}
\end{array}\right), \quad \hat{U} \hat{U}^{\dagger}=\hat{1}_{2 N} .
$$

Applying CC symmetry $\mathcal{C}_{+}$[Eq. (6.1)] to these $\mathrm{BCs}$ gives the relations

$$
\hat{u}_{h e}=\hat{u}_{e h}^{*}, \quad \hat{u}_{h h}=\hat{u}_{e e}^{*} .
$$

Choosing the matrices $\hat{u}_{e e}$ and $\hat{u}_{e h}$ as independent parameters, the unitarity of $\hat{U}$ reduces to the following constraints:

$$
\hat{u}_{e e} \hat{u}_{e e}^{\dagger}+\hat{u}_{e h} \hat{u}_{e h}^{\dagger}=\hat{1}_{N}, \quad \hat{u}_{e e} \hat{u}_{e h}^{\mathrm{T}}+\hat{u}_{e h} \hat{u}_{e e}^{\mathrm{T}}=\hat{0}_{N} .
$$

The equation (6.4) with Eq. (6.3) and the unitary matrix (6.5) satisfying the constraints (6.6) and (6.7) are thus the most general form of the $\mathrm{BCs}$ of a $\mathcal{C}_{+}$-symmetric system with a boundary, which describe all possible interfaces with all possible microscopic structures, and where the inaccessible region $x<0$ could be an insulator or a superconductor.

Explicitly resolving the constraints (6.7) for $N>1$ is not trivial and we do not attempt it here. Instead, we derive a subset of the general BCs, which we term normal-reflection BCs, that describe the boundary between a superconductor and an insulator, of interest in this work. For such an interface, there cannot be superconducting coupling between electrons and holes originating from it. Hence, the matrix $\hat{u}_{e h}=\hat{0}$ responsible for such processes must vanish. The remaining matrix $\hat{u}_{e e}=\hat{U}_{e e}$ of order $N$ is then a unitary matrix [Eq. (6.7)], describing scattering within the electron subspace only. The full scattering matrix is block-diagonal:

$$
\hat{U}=\left(\begin{array}{cc}
\hat{U}_{e e} & \hat{0} \\
\hat{0} & \hat{U}_{e e}^{*}
\end{array}\right), \quad \hat{U}_{e e} \hat{U}_{e e}^{\dagger}=\hat{1}_{N} .
$$

These are the normal-reflection BCs of the most general form, describing an arbitrary interface with an insulator. Note that for such normal-reflection BCs, the electron $j_{e+}(0)+$ $j_{e-}(0)=0$ and hole $j_{h+}(0)+j_{h-}(0)=0$ currents vanish independently.

Next, we analyze the bound states. As in the 1FS case (Sec. IV), we consider one-harmonic coordinate dependencies

$$
\Delta_{q}^{n}(x)=\Delta_{0}^{n} e^{i q^{n} x}, \quad \Delta_{0}^{n}=\left|\Delta_{0}^{n}\right| e^{-i \delta^{n}},
$$

with independent momenta $q^{n}$ of the pairing fields within each FS; such forms could help mitigate the effect of the Fermipoint mismatch. These explicit coordinate dependencies can be eliminated via the change of basis from $\hat{\psi}(x)$ to $\hat{\psi}^{\prime}(x)$ and $\hat{H}(\hat{p})$ to $\hat{H}^{\prime}(\hat{p})$, described by the same Eqs. (4.2)-(4.5) within each FS subspace.

Again, we look for the general solution to the Schrödinger equation

$$
\hat{H}^{\prime}(\hat{p}) \hat{\psi}^{\prime}(x)=\epsilon \hat{\psi}^{\prime}(x)
$$

that decays into the bulk. Since the FSs are not coupled by the Hamiltonian, there are independent particular solutions $\hat{\mathcal{X}}_{ \pm}^{n}(\epsilon) e^{i p_{ \pm}^{n}(\epsilon) x}$ for each FS $n$, with momentum solutions $p_{ \pm}^{n}(\epsilon)$ $[(5.1)]$ to the characteristic equation

$$
\operatorname{det}\left[\hat{H}^{\prime}(p)-\epsilon \hat{1}_{4 N}\right]=0
$$

and eigenvectors $\hat{\mathcal{X}}_{+}^{n}(\epsilon)$, which have the structure of Eq. (6.11) in the Gor'kov-Nambu subspace of the $n$th FS and are zero in the subspaces of other FSs. The general decaying solution

$$
\hat{\psi}^{\prime}(x)=\sum_{n=1}^{N}\left[c_{+}^{n} \hat{\mathcal{X}}_{+}^{n}(\epsilon) e^{i p_{+}^{n}(\epsilon) x}+c_{-}^{n} \hat{\mathcal{X}}_{-}^{n}(\epsilon) e^{i p_{-}^{n}(\epsilon) x}\right]
$$

is their linear combination with the free coefficients $c_{ \pm}^{n}$.

Inserting this general solution $\hat{\psi}(0)=\hat{\psi}^{\prime}(0)$ into the normal-reflection BCs [Eqs. (6.4) and (6.8)], we obtain the 
equations

$$
\hat{X}_{e+}(\epsilon) \hat{c}_{+}=\hat{U}_{e e} \hat{c}_{-}, \quad \hat{X}_{h+}(\epsilon) \hat{c}_{-}=\hat{U}_{e e}^{*} \hat{c}_{+}
$$

(note that velocity factors completely drop out again), where

$$
\hat{c}_{ \pm}=\left(\begin{array}{c}
c_{ \pm}^{1} \\
\ldots \\
c_{ \pm}^{N}
\end{array}\right)
$$

are the vectors of the free coefficients and

$$
\hat{X}_{v+}(\epsilon)=\operatorname{diag}\left(X_{v+}^{1}(\epsilon), \ldots, X_{v+}^{N}(\epsilon)\right)
$$

are diagonal matrices in the FS space, with $X_{v+}^{n}(\epsilon)$ being the factors (5.3) for each FS. From the scalar relation (5.4) for one $1 \mathrm{FS}$, the analogous relation follows for the diagonal matrices:

$$
\hat{X}_{h+}(\epsilon)=\hat{X}_{e+}^{*}(-\epsilon) \text {. }
$$

Excluding $\hat{c}_{+}$from Eq. (6.11) and using the unitarity of $\hat{U}_{e e}$, we arrive at the equation

$$
\hat{W}(\epsilon) \hat{c}_{-}=\hat{0}
$$

for $\hat{c}_{-}$, with

$$
\hat{W}(\epsilon)=\hat{U}_{e e}-\hat{X}_{e+}(\epsilon) \hat{U}_{e e}^{\mathrm{T}} \hat{X}_{e+}^{*}(-\epsilon) .
$$

A nontrivial solution for $\hat{c}_{-}$at some energy $\epsilon$ exists, if the matrix $\hat{W}(\epsilon)$ is degenerate. Hence, the energies of possible bound states are determined from the equation

$$
\operatorname{det} \hat{W}(\epsilon)=0 \text {. }
$$

Further analysis follows closely that of Ref. [45]; however, as we show, the result holds for a more general Hamiltonian (6.2) that does not assume $\mathcal{T}_{ \pm}$symmetries, and thus includes unequal velocities $v_{ \pm}^{n}$ of the right- and left-movers, Fermipoint mismatches $\varepsilon_{0}^{n}$, and coordinate dependencies (6.9) of the pairing fields.

Using the key relation (6.12) and the obvious relation $\hat{X}_{e+}(\epsilon) \hat{X}_{e+}^{*}(\epsilon)=\hat{1}$, we obtain the relation

$$
\hat{W}^{\mathrm{T}}(\epsilon)=-\hat{X}_{e+}^{*}(-\epsilon) \hat{W}(-\epsilon) \hat{X}_{e+}(\epsilon) .
$$

Taking the determinant of both sides at zero energy leads to the relation

$$
\operatorname{det} \hat{W}(0)=\operatorname{det}\left(-\hat{1}_{N}\right) \operatorname{det} \hat{W}(0)=(-1)^{N} \operatorname{det} \hat{W}(0) .
$$

Hence, for odd number $N$ of FSs, when $(-1)^{N}=-1$, the determinant necessarily vanishes at zero energy,

$$
\operatorname{det} \hat{W}(0)=0 \text {. }
$$

This means that there exists at least one zero-energy Majorana bound state, regardless of the parameters of the Hamiltonian [Eq. (6.2)] and of the unitary matrix $\hat{U}_{e e}$ [Eq. (6.8)] specifying the most general form of normal-reflection BCs. This proves the main claim in the case of any odd number $N$ of FSs. Note that for more than 1FS, there may generally be additional bound states that come in pairs of nonzero energies $\pm \varepsilon$ and are thus not protected by symmetry: upon varying the parameters, these pairs of bound states may disappear by merging with the bulk states. Also, at some values of parameters, their energies may turn to zero, $\varepsilon=0$, which will result in additional degenerate Majorana bound states of accidental nature.

\section{GENERALIZED QUANTUM-WIRE MODEL}

In this and the next sections, we demonstrate how instances of the general low-energy model [Eqs. (2.11) and (3.5)] for $1 \mathrm{FS}$ derived above purely from symmetry considerations and probability-current conservation principle arise from "microscopic" models [52] in the low-energy limit. We present the systematic procedure of deriving both the Hamiltonian and $\mathrm{BCs}$ of the low-energy model from the underlying microscopic model.

As the first example, we consider a model of a quantum wire, which has a few generalizations relative to that of Refs. [3,4]: an arbitrary direction of the Zeeman field and a general spin structure of the superconducting pairing field.

\section{A. Electron Hamiltonian and its bulk spectrum}

We consider a quantum-wire model for spinful electrons with the wave function

$$
\hat{\Psi}_{e}(x)=\left(\begin{array}{c}
\Psi_{e \uparrow}(x) \\
\Psi_{e \downarrow}(x)
\end{array}\right)
$$

described by the following electron Hamiltonian:

$$
\begin{gathered}
\hat{\mathcal{H}}_{e}(\hat{k})=\hat{\mathcal{H}}_{e 0}(\hat{k})-h_{z} \sigma_{z}, \\
\hat{\mathcal{H}}_{e 0}(\hat{k})=\left(\beta \hat{k}^{2}-\mu\right) \sigma_{0}-\alpha \hat{k} \sigma_{z}-h_{x} \sigma_{x}-h_{y} \sigma_{y} .
\end{gathered}
$$

Here $\hat{k}=-i \partial_{x}$ is the momentum operator, $\beta>0$ is the curvature coefficient of the quadratic term, $\alpha>0$ is the velocity coefficient of the linear spin-orbit term, $\mu$ is the chemical potential, $\sigma_{0}=\hat{1}$ is the unity matrix, and $\sigma_{\gamma}, \gamma=x, y, z$, are the spin Pauli matrices.

We assume that without the magnetic field the system (also the one with the boundary) possesses the reflection symmetry $\Sigma_{z}: z \rightarrow-z$ along the horizontal $z$ axis perpendicular to the wire, as shown in Fig. 1. This $z$ axis is also the direction of the average spin-orbit field $[\langle\mathbf{E}(\mathbf{r})\rangle \times \mathbf{k}]$, where the microscopic electric field $\mathbf{E}(\mathbf{r})$ is on average oriented along the vertical $y$ direction due to the broken reflection symmetry along it and assumed $\Sigma_{z}$ symmetry. Choosing this direction as the $z$ spin quantization axis gives the spin-orbit term $-\alpha \sigma_{z} \hat{k}$. Next, we consider the magnetic field $\mathbf{B}=\left(B_{x}, B_{y}, B_{z}\right)$ of arbitrary orientation. The total Zeeman term, assumed to have full spin rotational symmetry, reads $-h_{x} \sigma_{x}-h_{y} \sigma_{y}-h_{z} \sigma_{z}$, with $\mathbf{h}=\frac{g}{2} \mu_{B} \mathbf{B}$, where $\mu_{B}$ is the Bohr magneton.

As a result, the full electron Hamiltonian $\mathcal{H}_{e}(\hat{k})$ has axial spin rotation symmetry about the $z$ axis. Its bulk spectrum [Fig. 1(b)]

$$
\mathcal{E}_{e \pm}(k)=\beta k^{2}-\mu \pm \sqrt{\left(\alpha k+h_{z}\right)^{2}+h_{\perp}^{2}}
$$

depends only on the amplitude $h_{\perp}$, but not the angle $\phi_{\perp}$, of the part

$$
\left(h_{x}, h_{y}\right)=h_{\perp}\left(\cos \phi_{\perp}, \sin \phi_{\perp}\right)
$$

of the Zeeman field in the vertical $x y$ plane containing the wire. The $h_{z}$ Zeeman term causes the Fermi-point mismatch due to the asymmetry $\mathcal{E}_{e \pm}(k) \neq \mathcal{E}_{e \pm}(-k)$, which disfavors superconductivity, as discussed in Sec. IV.

For absent $h_{z}=0, \hat{\mathcal{H}}_{e 0}(\hat{k})$ is the electron Hamiltonian of the quantum-wire model introduced in Refs. [3,4]. As noticed 

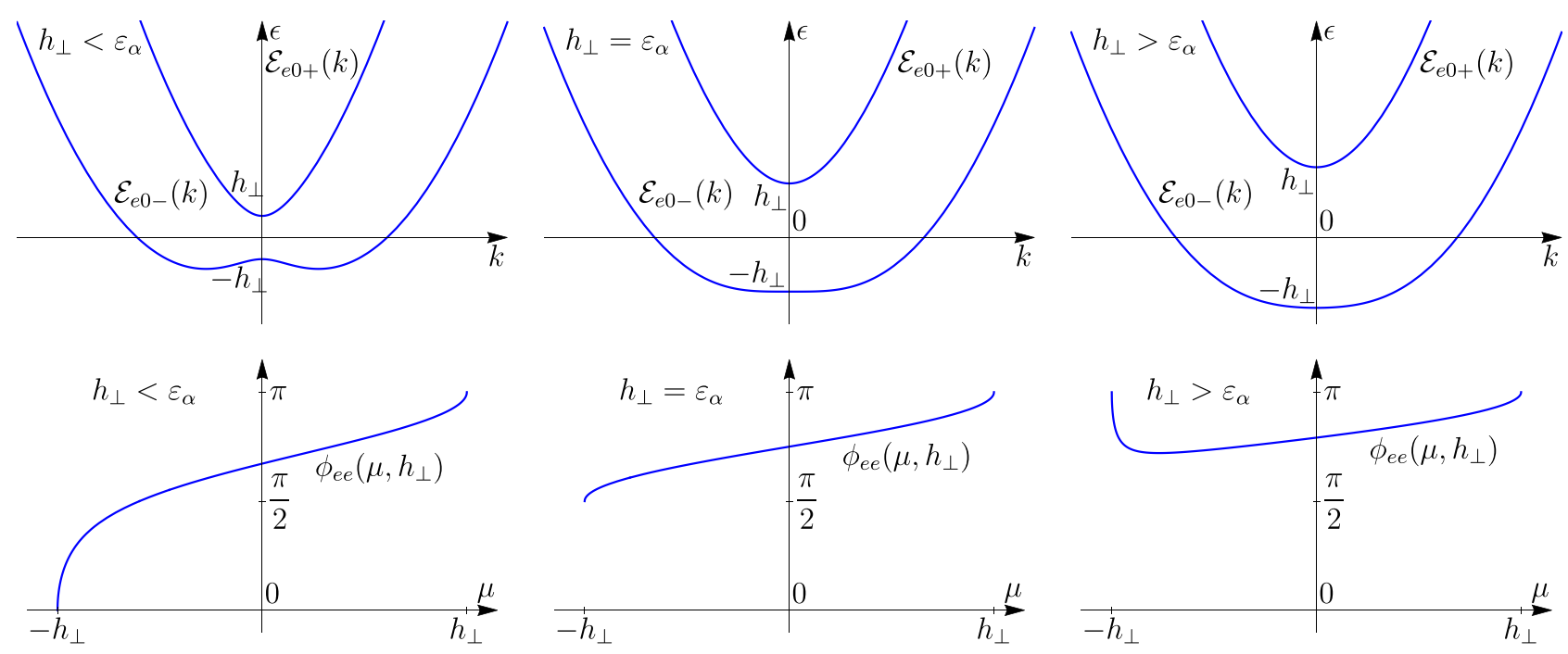

FIG. 8. Two different regimes $h_{\perp} \gtrless \varepsilon_{\alpha}$ of the behavior of the lower band $\mathcal{E}_{e 0-}(k)$ [Eq. (7.5)] of the Hamiltonian $\mathcal{H}_{e 0}(k)$ [Eq. (7.3)] of the quantum wire at $h_{z}=0$ and the borderline case $h_{\perp}=\varepsilon_{\alpha}$ between them. The upper row shows the spectra. The lower row shows the dependence of the scattering phase $\phi_{e e}\left(\mu, h_{\perp}\right)$ [Eq. (7.22)] in the low-energy normal-reflection boundary conditions [Eqs. (3.5) and (7.21)] on the chemical potential $\mu$ in each case.

in Refs. [45,47,48] and as we discuss in Appendix A 3, in this case, the electron system has an effective TR symmetry $\mathcal{T}_{e+}$ with $\mathcal{T}_{e+}^{2}=+1$, which prohibits the Fermi-point mismatch. The bulk spectrum of the electron Hamiltonian $\hat{\mathcal{H}}_{e 0}(k)$ without the $h_{z}$ term reads [Fig. 1(b)]

$$
\mathcal{E}_{e 0 \pm}(k)=\beta k^{2}-\mu \pm \sqrt{\alpha^{2} k^{2}+h_{\perp}^{2}}
$$

Whenever the $h_{\perp}$ Zeeman field is present, there is a range $\mu \in$ $\left(-h_{\perp}, h_{\perp}\right)$ of the chemical potential, where the system has 1FS, in which a Majorana bound state was originally predicted in Refs. [3,4]. This is the regime of interest in this work. Since we assume the effect of the Fermi-point mismatch small in the low-energy limit, we will take the $h_{z}$ Zeeman term into account perturbatively.

There are two regimes of the behavior of the lower band $\mathcal{E}_{e 0-}(k)$, see Fig. 8: (i) for $h_{\perp}<\varepsilon_{\alpha}$, where

$$
\varepsilon_{\alpha}=\frac{\alpha^{2}}{2 \beta}
$$

is the crossover energy scale, at which the spin-orbit $\sim \alpha k$ and quadratic $\sim \beta k^{2}$ terms become comparable, the lower band has a maximum at $k=0$ and two minima; (ii) for $h_{\perp}>\varepsilon_{\alpha}$, the lower band has only a minimum at $k=0$. In either regime $h_{\perp} \gtrless \varepsilon_{\alpha}$, for $\mu \in\left(-h_{\perp}, h_{\perp}\right)$, the Fermi level crosses only the lower band $\mathcal{E}_{e 0-}(k)$ twice, at the Fermi points $k= \pm k_{0}$, where the Fermi momentum

$$
k_{0}=\sqrt{\left(s+\mu+\varepsilon_{\alpha}\right) / \beta}, \quad s=\sqrt{h_{\perp}^{2}+2 \mu \varepsilon_{\alpha}+\varepsilon_{\alpha}^{2}},
$$

is obtained from $\mathcal{E}_{e 0-}(k)=0$. The Fermi points are at opposite momenta $k= \pm k_{0}$ in the absence of the $h_{z}$ Zeeman field, which is the consequence of the effective TR symmetry $\mathcal{T}_{e+}$, as we discuss in Appendix A 3.
The normalized bulk eigenstates of the Hamiltonian $\hat{\mathcal{H}}_{e 0}(k)$ for the lower band $\mathcal{E}_{e 0-}(k)$ are

$$
\begin{aligned}
\hat{\chi}_{e}(k) & =\left(\begin{array}{l}
\chi_{e \uparrow}(k) \\
\chi_{e \downarrow}(k)
\end{array}\right) \\
& =\frac{1}{\sqrt{N(k)}}\left(\begin{array}{c}
e^{-\frac{i}{2} \phi_{\perp}} h_{\perp} \\
e^{\frac{i}{2} \phi_{\perp}}\left(\sqrt{\alpha^{2} k^{2}+h_{\perp}^{2}}-\alpha k\right)
\end{array}\right), \\
N(k) & =2 \sqrt{\alpha^{2} k^{2}+h_{\perp}^{2}}\left(\sqrt{\alpha^{2} k^{2}+h_{\perp}^{2}}-\alpha k\right),
\end{aligned}
$$

which will be used in the next section.

\section{B. General form of the superconducting pairing field}

The general BdG Hamiltonian built from the electron Hamiltonian (7.2) of the quantum wire has the standard block structure

$$
\hat{\mathcal{H}}(\hat{k})=\left(\begin{array}{cc}
\hat{\mathcal{H}}_{e}(\hat{k}) & \hat{\Delta}[x, \cdot] \\
\Delta^{\dagger}[x, \cdot] & -\hat{\mathcal{H}}_{e}^{\mathrm{T}}(-\hat{k})
\end{array}\right)
$$

for the $\mathrm{BdG}$ wave function

$$
\hat{\Psi}(x)=\left(\begin{array}{c}
\hat{\Psi}_{e}(x) \\
\hat{\Psi}_{h}(x)
\end{array}\right), \quad \hat{\Psi}_{h}(x)=\left(\begin{array}{c}
\Psi_{h \uparrow}(x) \\
\Psi_{h \downarrow}(x)
\end{array}\right) .
$$

The charge-conjugation operation reads

$$
\mathcal{C}_{+} \hat{\Psi}(x)=\hat{C}_{+} \hat{\Psi}^{*}(x), \quad \hat{C}_{+}=\tau_{x} \otimes \sigma_{0} .
$$

As another generalization of the model proposed in Refs. [3,4], we consider the most general form of the superconducting pairing field, described by the nonlocal integral operator, acting on the wave function as

$$
\begin{aligned}
& \hat{\Delta}\left[x, \hat{\Psi}_{h}\right]=\int d x^{\prime} \hat{\Delta}\left(x, x^{\prime}\right) \hat{\Psi}_{h}\left(x^{\prime}\right), \\
& \hat{\Delta}^{\dagger}\left[x, \hat{\Psi}_{e}\right]=\int d x^{\prime} \hat{\Delta}^{\dagger}\left(x^{\prime}, x\right) \hat{\Psi}_{e}\left(x^{\prime}\right) .
\end{aligned}
$$


Its kernel

$$
\hat{\Delta}\left(x, x^{\prime}\right)=\sum_{\gamma=0, x, y, z} \Delta_{\gamma}\left(x, x^{\prime}\right) \sigma_{\gamma} i \sigma_{y}
$$

is a matrix function in the spin space consisting of the singlet $\gamma=0$ and triplet $\gamma=x, y, z$ contributions. In Refs. [3,4], only the singlet term was considered.

As enforced by $\mathrm{CC}$ symmetry, the pairing-field operator is antisymmetric, $\hat{\Delta}\left(x, x^{\prime}\right)=-\hat{\Delta}^{\mathrm{T}}\left(x^{\prime}, x\right)$. As a result, the scalar kernels $\Delta_{\gamma}\left(x, x^{\prime}\right)$ of the singlet and triplet components are symmetric, $\Delta_{0}\left(x, x^{\prime}\right)=\Delta_{0}\left(x^{\prime}, x\right)$, and antisymmetric, $\Delta_{x, y, z}\left(x, x^{\prime}\right)=-\Delta_{x, y, z}\left(x^{\prime}, x\right)$, respectively. For this reason, the kernels of the triplet components must necessarily be nonlocal to be nonzero.

\section{Derivation of the low-energy model}

We now derive the low-energy model from the above microscopic model of the quantum wire in the 1FS regime, $\mu \in\left(-h_{\perp}, h_{\perp}\right)$, which describes the limit of energies $\epsilon$ close to the Fermi level. We assume the $h_{z}$ Zeeman field and the superconducting pairing field (7.11) small and take them into account perturbatively.

For the derivation of the low-energy model, we consider the BdG wave function [Eq. (7.10)]

$$
\begin{aligned}
\hat{\Psi}(x)= & \psi_{e+}(x)\left(\begin{array}{c}
\hat{\chi}_{e}\left(+k_{0}\right) \\
\hat{0}
\end{array}\right) e^{+i k_{0} x}+\psi_{e-}(x)\left(\begin{array}{c}
\hat{\chi}_{e}\left(-k_{0}\right) \\
\hat{0}
\end{array}\right) e^{-i k_{0} x} \\
& +\psi_{h-}(x)\left(\begin{array}{c}
\hat{0} \\
\hat{\chi}_{h}\left(+k_{0}\right)
\end{array}\right) e^{+i k_{0} x}+\psi_{h+}(x)\left(\begin{array}{c}
\hat{0} \\
\hat{\chi}_{h}\left(-k_{0}\right)
\end{array}\right) e^{-i k_{0} x}
\end{aligned}
$$

as a linear combination of the bulk plane-wave eigenstates at the Fermi level $\epsilon=0$ of the Hamiltonian

$$
\hat{\mathcal{H}}_{0}(\hat{k})=\left(\begin{array}{cc}
\hat{\mathcal{H}}_{e 0}(\hat{k}) & \hat{0} \\
\hat{0} & -\hat{\mathcal{H}}_{e 0}^{\mathrm{T}}(-\hat{k})
\end{array}\right)
$$

with the neglected $h_{z}$ Zeeman term and pairing field (7.11). There are four such plane-wave bulk eigenstates. The first and second ones in Eq. (7.12) are pure electron solutions for the lower band $\mathcal{E}_{e 0-}(k)$ [Eq. (7.5)] at the Fermi points $\pm k_{0}, \hat{\mathcal{H}}_{e 0}\left( \pm k_{0}\right) \hat{\chi}_{e}\left( \pm k_{0}\right)=\hat{0}$, with $\hat{\chi}_{e}\left( \pm k_{0}\right)$ given by Eq. (7.8). The third and fourth ones in Eq. (7.12) with $\hat{\chi}_{h}\left( \pm k_{0}\right)=$ $\hat{\chi}_{e}^{*}\left(\mp k_{0}\right)$ are pure hole solutions at the Fermi points $\pm k_{0}$, $-\hat{\mathcal{H}}_{e 0}^{\mathrm{T}}\left(\mp k_{0}\right) \hat{\chi}_{h}\left( \pm k_{0}\right)=\hat{0}$, and are charge conjugates of the former electron solutions. The coefficients $\psi_{e \pm}(x), \psi_{h \pm}(x)$ of the linear combination represent the components of the low-energy BdG wave function $\hat{\psi}(x)$ [Eq. (2.5)], whose slow coordinate dependence compared to the scales set by $\mu, h_{\perp}$, $\varepsilon_{\alpha}$ is meant to account for the deviation of the energy $\epsilon$ from the Fermi level and for the presence of the small $h_{z}$ Zeeman term and pairing field.

Inserting the wave function (7.12) into the full BdG Hamiltonian $\hat{\mathcal{H}}(\hat{k})$ [Eq. (7.9)] with the pairing field and $h_{z}$ Zeeman term and keeping the terms up to the linear order in momentum $\hat{p} \hat{\psi}(x)$ acting on the low-energy wave function, we obtain the low-energy BdG Hamiltonian $\hat{H}(\hat{p})$ of the form (2.11) for $\hat{\psi}(x)$, with equal velocities $v_{ \pm}=v$,

$$
\begin{aligned}
v & =\hat{\chi}_{e}^{\dagger}\left(k_{0}\right)\left(2 \beta k_{0} \sigma_{0}-\alpha \sigma_{z}\right) \hat{\chi}_{e}\left(k_{0}\right) \\
& =2 \beta k_{0}-\frac{\alpha^{2} k_{0}}{\sqrt{\alpha^{2} k_{0}^{2}+h_{\perp}^{2}}}
\end{aligned}
$$

the Fermi-point mismatch [53] energy [Eq. (2.1)]

$$
\varepsilon_{0}=-\hat{\chi}_{e}^{\dagger}\left(k_{0}\right) h_{z} \sigma_{z} \hat{\chi}_{e}\left(k_{0}\right)=-\frac{\alpha k_{0}}{\sqrt{\alpha^{2} k_{0}^{2}+h_{\perp}^{2}}} h_{z}
$$

due to the $h_{z}$ Zeeman field, and the low-energy pairing field $\hat{\Delta}(x)=i \tau_{y} \Delta(x)$ with

$$
\begin{aligned}
\Delta(x)= & \hat{\chi}_{e}^{\dagger}\left(k_{0}\right) \hat{\Delta}\left(x, k_{0}\right) \hat{\chi}_{h}\left(k_{0}\right) \\
= & \frac{\alpha k_{0}}{\sqrt{\alpha^{2} k_{0}^{2}+h_{\perp}^{2}}} \Delta_{0}\left(x, k_{0}\right)+\Delta_{z}\left(x ; k_{0}\right) \\
& +\frac{i h_{\perp}}{\sqrt{\alpha^{2} k_{0}^{2}+h_{\perp}^{2}}}\left[-\Delta_{x}\left(x ; k_{0}\right) \sin \phi_{\perp}\right. \\
& \left.+\Delta_{y}\left(x ; k_{0}\right) \cos \phi_{\perp}\right],
\end{aligned}
$$

expressed in terms of the parameters of the microscopic quantum-wire model. In Eq. (7.16),

$$
\Delta_{\gamma}(x ; k)=\int d x^{\prime \prime} e^{-i k x^{\prime \prime}} \Delta_{\gamma}\left(x+\frac{1}{2} x^{\prime \prime}, x-\frac{1}{2} x^{\prime \prime}\right)
$$

are the Wigner transforms of the pairing-field kernels.

We observe that in the limit of the 1FS low-energy model, all spin components $\Delta_{0, x, y, z}\left(x ; k_{0}\right)$ of the microscopic pairing field (7.11) do reduce to just one unique, antisymmetric form $\hat{\Delta}(x)=i \tau_{y} \Delta(x)$ of the low-energy pairing field in Eqs. (2.10) and (2.11), while the spin-orbit and $h_{\perp}$ Zeeman effects control whether a given component contributes or not. The spin-singlet component $\Delta_{0}\left(x ; k_{0}\right)$ contributes to $\Delta(x)$ only if the spin-orbit effect is present, $\alpha>0$. The spintriplet components $\Delta_{x, y, z}\left(x ; k_{0}\right)$ contribute to $\Delta(x)$ whether the spin-orbit effect is present or not. The $\Delta_{x, y}\left(x ; k_{0}\right)$ components contribute only when $h_{\perp}$ is present; interestingly, one may recognize that the combination $-\Delta_{x}\left(x ; k_{0}\right) \sin \phi_{\perp}+$ $\Delta_{y}\left(x ; k_{0}\right) \cos \phi_{\perp}$ in which they enter $\Delta(x)$ is the projection of the vector $\left(\Delta_{x}\left(x ; k_{0}\right), \Delta_{y}\left(x ; k_{0}\right)\right)$ on the direction in the $x y$ plane perpendicular to the vector $\left(h_{x}, h_{y}\right)$. The $h_{\perp}$ Zeeman field is per se not necessary for $\Delta_{0, z}\left(x ; k_{0}\right)$ to contribute to $\Delta(x)$; however, without it, the 1FS regime is not possible in this system, since the bands $\mathcal{E}_{e 0 \pm}(k)$ [Eq. (7.5)] would then have a crossing point at $k=0$.

\section{Boundary conditions}

The normal- and Andreev-reflection BCs (3.5) and (3.6) derived in Sec. III represent the two families of all possible BCs for the general low-energy model that satisfy only the current-conservation principle and $\mathcal{C}_{+}$symmetry. When the underlying microscopic model with its boundary is fully specified, the corresponding BCs of its low-energy model are an instance of these general BCs. We now explicitly demonstrate this point by deriving the corresponding $\mathrm{BCs}$ for the lowenergy wave function $\hat{\psi}(x)$ [Eq. (2.5)] from the quantum wire model. Variants of such systematic derivation procedure have 
already been demonstrated for other low-energy models, e.g., for graphene lattice [38], Luttinger semimetal [41], and quantum anomalous Hall system [42]. This derivation procedure of specific BCs for the low-energy model from an underlying microscopic model should not be conflated with the derivation procedure of the general BCs performed in Sec. III, based on the current-conservation principle and symmetries.

We assume that the quantum wire occupies the region $x>$ 0 (Fig. 1) and consider the hard-wall BCs [54]

$$
\hat{\Psi}_{e}(x=0)=\hat{0}
$$

for the electron part of the BdG wave function (7.1), which nullify it at the boundary $x=0$. The corresponding BCs for the hole part are obtained by CC operation, which in this case leads to the hard-wall BCs

$$
\hat{\Psi}_{h}(x=0)=\hat{0}
$$

as well.

For the derivation of the $\mathrm{BCs}$, to the leading order, one may consider the Hamiltonian $\hat{\mathcal{H}}_{0}(\hat{k})$ [Eq. (7.13)], with the neglected small pairing field and $h_{z}$ Zeeman field, instead of the full microscopic Hamiltonian $\hat{\mathcal{H}}(\hat{k})$ [Eq. (7.9)] and consider the energy right at the Fermi level $\epsilon=0$. Once the pairing field is neglected, electron $\hat{\Psi}_{e}(x)$ and hole $\hat{\Psi}_{h}(x)$ parts of BdG wave function (7.10) are decoupled and one may consider them separately. This already guarantees that the resulting low-energy BCs will have the form of the normal-reflection BCs (3.5).

So, we look for a general solution to the Schrödinger equation $\hat{\mathcal{H}}_{e 0}(\hat{k}) \hat{\Psi}_{e}(x)=\hat{0}$ for the electron part of the wave function that does not grow into the bulk (as $x \rightarrow+\infty$ ). The general solution is a linear combination of particular solutions with the coordinate dependence $\propto e^{i k x}$ with generally complex momenta $k$. The latter are determined from the characteristic equation

$$
\operatorname{det} \hat{\mathcal{H}}_{e 0}(k)=\left(\beta k^{2}-\mu\right)^{2}-\alpha^{2} k^{2}-h_{\perp}^{2}=0,
$$

which has four solutions. In the regime $\mu \in\left(-h_{\perp}, h_{\perp}\right)$ of $1 \mathrm{FS}$ we consider, two of these solutions $k= \pm k_{0}$ are, as expected, the real Fermi-point momenta [Eq. (7.7)], and the corresponding eigenvectors are the bulk states $\hat{\chi}_{e}\left( \pm k_{0}\right)$ [Eq. (7.8)]. The other two solutions $k= \pm i \varkappa, \varkappa=\sqrt{\left(s-\mu-\varepsilon_{\alpha}\right) / \beta}$, are imaginary, with the wave functions $\propto e^{\mp \varkappa x}$ decaying and growing into the bulk, respectively. The eigenvector of the decaying solution $k=i \varkappa$ reads

$$
\hat{\chi}_{e 0}=\left(\begin{array}{c}
e^{-\frac{i}{2} \phi_{\perp}} h_{\perp} \\
e^{\frac{i}{2} \phi_{\perp}}\left[\varepsilon_{\alpha}-s+i \sqrt{2 \varepsilon_{\alpha}\left(s-\mu-\varepsilon_{\alpha}\right)}\right]
\end{array}\right) .
$$

Hence, the general solution that does not grow into the bulk reads

$\hat{\Psi}_{e}(x)=\psi_{e+} \hat{\chi}_{e}\left(+k_{0}\right) e^{+i k_{0} x}+\psi_{e} \hat{\chi}_{e}\left(-k_{0}\right) e^{-i k_{0} x}+C \hat{\chi}_{e 0} e^{-\varkappa x}$.

It consists of two bulk plane-wave states and one decaying solution, entering with arbitrary coefficients $\psi_{e \pm}$ and $C$. Inserting this form into the BCs (7.18), we obtain two linear relations

$$
\begin{aligned}
& \psi_{e+\chi_{e \uparrow}}\left(+k_{0}\right)+\psi_{e-} \chi_{e \uparrow}\left(-k_{0}\right)+C \chi_{e 0 \uparrow}=0, \\
& \psi_{e+\chi_{e \downarrow}}\left(+k_{0}\right)+\psi_{e-\chi_{e \downarrow}}\left(-k_{0}\right)+C \chi_{e 0 \downarrow}=0
\end{aligned}
$$

for the three coefficients $\psi_{e+}, \psi_{e_{-}}, C$. Excluding $C$, we obtain one relation

$$
\psi_{e+}=U_{e e}\left(\mu, h_{\perp}\right) \psi_{e-}
$$

for the two coefficients $\psi_{e+}$ and $\psi_{e-}$, where

$$
\begin{aligned}
U_{e e}\left(\mu, h_{\perp}\right) & =e^{-i \phi_{e e}\left(\mu, h_{\perp}\right)} \\
& =-\frac{\chi_{e \uparrow}\left(-k_{0}\right) \chi_{e 0 \downarrow}-\chi_{e \downarrow}\left(-k_{0}\right) \chi_{e 0 \uparrow}}{\chi_{e \uparrow}\left(+k_{0}\right) \chi_{e 0 \downarrow}-\chi_{e \downarrow}\left(+k_{0}\right) \chi_{e 0 \uparrow}} .
\end{aligned}
$$

The same relation will hold, to leading order, for the coordinate-dependent components of the electron part of the low-energy BdG wave function (2.5) at the boundary, $\psi_{e \pm} \rightarrow$ $\psi_{e \pm}(x=0)$. Hence, the relation (7.21) represents the BC for it and $U_{e e}\left(\mu, h_{\perp}\right)$ is indeed the phase factor therein. The BC for the hole part $\hat{\psi}_{h}(x)$ of the low-energy BdG wave function can be obtained analogously. We thus find that indeed the $\mathrm{BCs}$ for the low-energy model derived from the quantum-wire model with the hard-wall BCs (7.18) and (7.19) have the form (3.5) of the general normal-reflection BCs. Note that taking the decaying solution $\propto e^{-\varkappa x}$ into account in Eq. (7.20) is essential for deriving the low-energy BCs.

The phase factor $U_{e e}\left(\mu, h_{\perp}\right)$ is a dimensionless function of the parameters of the microscopic model, the chemical potential $\mu$ and the $h_{\perp}$ Zeeman field relative to the spin-orbit scale $\varepsilon_{\alpha}$ [Eq. (7.6)]. In Fig. 8, we plot the dependence of the scattering phase $\phi_{e e}\left(\mu, h_{\perp}\right)$, defined in Eq. (7.22), on the chemical potential $\mu$. The dependence differs qualitatively in the two regimes $h_{\perp} \gtrless \varepsilon_{\alpha}$ of the behavior of the lower band $\mathcal{E}_{e 0-}(k)$ [Eq. (7.5)]. At the upper bound $\mu=h_{\perp}$ of the 1FS range $\mu \in\left(-h_{\perp}, h_{\perp}\right)$,

$$
U_{e e}\left(\mu=h_{\perp}, h_{\perp} \gtrless \varepsilon_{\alpha}\right)=-1
$$

in both regimes. At its lower bound $\mu=-h_{\perp}$,

$$
U_{e e}\left(\mu=-h_{\perp}, h_{\perp} \gtrless \varepsilon_{\alpha}\right)=\mp 1 .
$$

Accordingly, for $h_{\perp}<\varepsilon_{\alpha}$, the phase $\phi_{e e}\left(\mu, h_{\perp}\right)$ monotonically increases from 0 to $\pi$ as $\mu$ spans $\left(-h_{\perp}, h_{\perp}\right)$. For $h_{\perp}>$ $\varepsilon_{\alpha}, \phi_{e e}\left(\mu, h_{\perp}\right)$ has a minimum. In the borderline case $h_{\perp}=\varepsilon_{\alpha}$,

$$
U_{e e}\left(\mu=-h_{\perp}, h_{\perp}=\varepsilon_{\alpha}\right)=-i,
$$

and $\phi_{e e}\left(\mu, h_{\perp}=\varepsilon_{\alpha}\right)$ monotonically increases from $\pi / 2$ to $\pi$ as $\mu$ spans $\left(-h_{\perp}, h_{\perp}\right)$.

\section{EDGE OF A QUANTUM SPIN HALL SYSTEM}

In this section, we present another demonstration of the derivation of the low-energy model for 1FS with its BCs, this time from the model for the edge of the quantum spin Hall system, considered in Ref. [2]. For simplicity, we assume that the Fermi level is close to the crossing point of the counterpropagating edge states, so that the normal-state electron Hamiltonian for the two-component electron wave function

$$
\hat{\Psi}_{e}(x)=\left(\begin{array}{l}
\Psi_{e+}(x) \\
\Psi_{e-}(x)
\end{array}\right)
$$

can already be taken linear in momentum, in the form

$$
\hat{\mathcal{H}}_{e}(\hat{k})=\left(\begin{array}{cc}
v \hat{k}-\mu & 0 \\
0 & -v \hat{k}-\mu
\end{array}\right), \quad x>0 .
$$


This Hamiltonian satisfies TR symmetry $\mathcal{T}_{e-}$ [Eq. (A3)] with $\hat{T}_{e-}=i \tau_{y}$ and, as discussed in the Appendix, the edge states cannot be confined via backscattering without breaking it. One way to confine the edge states of a quantum spin Hall system is, as per the proposal of Ref. [2], by coupling it to a magnetic material. The effect of the magnetic material, which we assume to be placed in the region $x<0$, can be modeled by additional terms $m_{x, y}$ in the Hamiltonian (8.1)

$$
\hat{\mathcal{H}}_{e}(\hat{k})=\left(\begin{array}{cc}
v \hat{k}-\mu & m_{x}-i m_{y} \\
m_{x}+i m_{y} & -v \hat{k}-\mu
\end{array}\right), \quad x<0 .
$$

These terms open up a gap around the crossing point for energies $\epsilon+\mu \in\left(-m_{\perp}, m_{\perp}\right), m_{\perp}=\sqrt{m_{x}^{2}+m_{y}^{2}}$.

For the derivation of the low-energy model describing the energies $\epsilon$ close to the Fermi level, we consider the electron wave function

$$
\hat{\Psi}_{e}(x)=\left(\begin{array}{l}
\psi_{e+}(x) e^{+i k_{0} x} \\
\psi_{e-}(x) e^{-i k_{0} x}
\end{array}\right), \quad x>0,
$$

as expansion in terms of the eigenstates at the Fermi level $\epsilon=0 ; k_{0}=\mu / v$ is the Fermi momentum. The coordinatedependent expansion coefficients $\psi_{e \pm}(x)$ make up the lowenergy wave function (2.3), which varies over the spatial scales much exceeding $v / \mu$ and $v / m_{\perp}$ and is defined only in the region $x>0$. The Hamiltonian for it in that region has the form $\hat{H}_{e}^{\mathcal{T}_{e-}}(\hat{p})$ [Eq. (A5)].

Due to the gap opening in the region $x<0$, the wave function $\hat{\Psi}_{e}(x)$ at energies $\epsilon+\mu \in(-m, m)$ will decay as $x \rightarrow-\infty$ over a microscopic scale $v / \mu, v / m_{\perp}$. For the lowenergy wave function $\hat{\psi}_{e}(x)$, this will result in an effective BC at $x=0$.

Again, as in Sec. VIID, for the sake of deriving the BC, it is sufficient to consider the energy $\epsilon=0$ right at the Fermi level $\mu \in\left(-m_{\perp}, m_{\perp}\right)$. We construct a general solution to the Schrödinger equation $\hat{\mathcal{H}}_{e}(\hat{k}) \hat{\Psi}_{e}(x)=\hat{0}$ that decays into the magnetic region (as $x \rightarrow-\infty$ ) and does not grow into the bulk (as $x \rightarrow+\infty$ ). In the region $x>0$, the general solution

$$
\hat{\Psi}_{e}(x)=\left(\begin{array}{l}
\psi_{e+} e^{+i k_{0} x} \\
\psi_{e-} e^{-i k_{0} x}
\end{array}\right), \quad x>0,
$$

with constant coefficients $\psi_{e \pm}$, consists only of the particular solutions representing the low-energy wave function.

In the region $x<0$, the solution reads

$$
\hat{\Psi}_{e}(x)=C\left(i \sqrt{m_{\perp}^{2}-\mu^{2}}+\mu\right) e^{\frac{\sqrt{m_{\perp}^{2}-\mu^{2}}}{v} x}, \quad x<0,
$$

with $m=m_{x}-i m_{y}$ and an arbitrary coefficient $C$.

As follows from the differential properties of the Hamiltonian [Eqs. (8.1) and (8.2)], the solution to the Schrödinger equation must be continuous at $x=0$, i.e., $\hat{\Psi}_{e}(x=+0)=$ $\hat{\Psi}_{e}(x=-0)$. This leads to two relations

$$
\psi_{e+}=C m, \quad \psi_{e-}=C\left(i \sqrt{m_{\perp}^{2}-\mu^{2}}+\mu\right)
$$

for the three coefficients $\psi_{e \pm}$ and $C$. Excluding the coefficient $C$ from these equations, we obtain a relation

$$
\psi_{e+}=U_{e e} \psi_{e-}
$$

with

$$
U_{e e}=\frac{m}{i \sqrt{m_{\perp}^{2}-\mu^{2}}+\mu} .
$$

Again, the relation (8.3) represents the $\mathrm{BC}$ for the low-energy electron wave function and $U_{e e}$ is the phase factor therein. The $\mathrm{BC}$ for the hole part $\hat{\psi}_{h}(x)$ of the low-energy BdG wave function can be obtained analogously. We thus find that indeed the BCs for the low-energy model derived from the model of the edge of the quantum spin Hall system coupled to a magnetic material have the form (3.5) of the general normal-reflection BCs.

\section{LOW-ENERGY SYMMETRY-BASED APPROACH AND TOPOLOGY, OUTLOOK}

The main physical finding of this work and its practical implications have already been formulated and discussed in Secs. I and V. In this concluding section, we briefly discuss the relation of the employed theoretical formalism to the topological aspect of the system.

The low-energy symmetry-based formalism for studying the bound states in topological systems consists of two stages: (i) deriving the low-energy model of the most general form, whose Hamiltonian and BCs are subject only to symmetries and the fundamental principle of probability-current conservation; (ii) calculating and exploring the corresponding bound-state structure of the model. As such, remarkably, this formalism allows one to obtain and explore generic boundstate structures of topological systems without ever explicitly invoking the notion of topology. Nonetheless, the so-obtained bound-state structures will, of course, be in accord with the topological properties of the systems. For the particular class of systems studied in this paper, quasi-1D superconductors with odd number of FSs, interfaced with a vacuum or an insulator, the obtained ever-present Majorana bound state indeed most likely has topological origin. The $\epsilon=0$ bound states are topologically protected under $\mathrm{CC}$ symmetry $\mathcal{C}_{+}$, i.e., cannot be removed without breaking the symmetry or closing the bulk gap. In topological systems, bound states are anticipated based on the concept of bulk-boundary correspondence [31], which, however, to the best of our knowledge, remains unproven at the required level of rigor. Regardless, even if such proof is possible, the low-energy approach has apparent advantages in that it delivers the generic bound-state structure, which can be explored in an explicit fashion. As such, the formalism can be used not only to confirm or illustrate topological concepts, but rather, to test them and possibly discover new features.

This low-energy symmetry-based formalism is completely general and applicable to a multitude of other systems (as long as they allow for a well-defined low-energy limit). It has previously been applied to 2D chiral-symmetric semimetals [41] and quantum anomalous Hall systems (Chern insulators) in the vicinity of the topological phase transition [42]. Regarding superconductors, possible further extensions of the present work could be exploring the meaning and possible physical realizations of Andreev-reflection BCs for 1FS and extending the approach to higher dimensions. The low-energy model for two Fermi surfaces should, in particular, be useful for studying 
bound states in superconductors with magnetic interfaces or magnetic scatterers [55,56].

\section{RELATION TO PREVIOUS WORK}

Recently Ref. [45] came out, where a similar main conclusion about the existence of Majorana bound states was reached using a similar low-energy model. The main differences between our work and Ref. [45] are as follows. We derive the low-energy model based purely on CC symmetry and the current conservation principle, thereby proving that it is of the most general form, whereas in Ref. [45], neither CC symmetry nor the current conservation principle were addressed. The normal-reflection BCs were not derived, but rather postulated phenomenologically, and the Andreevreflection BCs for the case of 1FS did not arise in Ref. [45] at all. Further, the effective TR symmetry $\mathcal{T}_{e+}$ of the electron Hamiltonian was assumed necessary in Ref. [45], which prohibited the Fermi-point mismatch. We take the Fermi-point mismatch into account (as well as the coordinate dependence of the pairing field that helps mitigate it) and demonstrate that Majorana bound states persist in its presence, which is an important finding for practical applications.

\section{ACKNOWLEDGMENTS}

M.K. acknowledges financial support by DFG Grant No. KH 461/1-1. E.M.H. and B.T. acknowledge funding by the Deutsche Forschungsgemeinschaft (DFG; German Research Foundation) through SFB 1170, Project ID 258499086, through Grant No. HA 5893/4-1 within SPP 1666, and through the Würzburg-Dresden Cluster of Excellence on Complexity and Topology in Quantum Matter, ct.qmat (EXC 2147, Project ID 390858490), as well as by the ENB Graduate School on Topological Insulators. The work of F.S.B. was partially funded by Spanish Ministerio de Ciencia, Innovacion, y Universidades (MICINN) [Projects No. FIS2017-82804-P and No. PID2020-114252GB-I00 (SPIRIT)], and by Grupos Consolidados UPV/EHU del Gobierno Vasco (Grant No. IT1249-19).

\section{APPENDIX: TIME-REVERSAL SYMMETRIES}

In this Appendix, we consider the effects of possible additional TR symmetries $\mathcal{T}_{ \pm}$of the low-energy model for 1FS.

\section{Time-reversal symmetries of the Hamiltonian}

When introduced formally, a TR symmetry of a Hamiltonian means that there exists an antiunitary TR operation

$$
\mathcal{T}_{ \pm}=\hat{T}_{ \pm} \mathcal{K}, \quad \mathcal{T}_{ \pm} \hat{\psi}(x)=\hat{T}_{ \pm} \hat{\psi}^{*}(x),
$$

under which the Hamiltonian remains invariant,

$$
\hat{T}_{ \pm}[\hat{H}(\hat{p})]^{*} \hat{T}_{ \pm}^{\dagger}=\hat{H}(\hat{p}),
$$

with $\hat{p}^{*}=-\hat{p}$. Here, $\hat{T}_{ \pm}$are unitary matrices. Two different types $\mathcal{T}_{ \pm}$of TR operation are possible, squaring to either plus or minus unity,

$$
\mathcal{T}_{ \pm}^{2}=\hat{T}_{ \pm} \hat{T}_{ \pm}^{*}= \pm \hat{1}
$$

which we label accordingly with \pm .
For a spinful electron system, the actual TR operation is of the type $\mathcal{T}_{-}$. However, an effective $\mathcal{T}_{+}$symmetry could also be present in a spinful electron system, which can arise as a combination of the actual $\mathcal{T}_{-}$and some spatial operation, as is the case, e.g., for the quantum-wire system; see Fig. 1 and Appendix A 3.

We construct the most general forms of the $\mathcal{T}_{ \pm}$operations for the low-energy Hamiltonian $\hat{H}(\hat{p})$ [Eq. (2.11)]. The TR operation, whether $\mathcal{T}_{+}$or $\mathcal{T}_{-}$, interchanges the right- and leftmoving electron states $\psi_{e \pm}(x)$. The most general forms of the TR operations $\mathcal{T}_{e \pm}=\hat{T}_{e \pm} \mathcal{K}$ acting only on the electron part $\hat{\psi}_{e}(x)$ of the BdG wave function (2.5) are

$$
\begin{gathered}
\hat{T}_{e+}=\tau_{x}, \quad \hat{T}_{e-}=i \tau_{y}, \\
\mathcal{T}_{e \pm} \hat{\psi}_{e}(x)=\left(\begin{array}{c}
\psi_{e-}^{*}(x) \\
\pm \psi_{e+}^{*}(x)
\end{array}\right) .
\end{gathered}
$$

The overall phase factors of the operations can be chosen arbitrarily. For the $\mathcal{T}_{e-}$ operation, the electron states $\psi_{e \pm}(x)$ form a Kramers pair.

Either of these TR symmetries $\mathcal{T}_{e \pm}$ (to avoid confusion, here and below, we mean one of the operations at a time, but join them into one formula) restricts the form of the electron Hamiltonian $\hat{H}_{e}(\hat{p})$ [Eq. (2.2)] to

$$
\hat{H}_{e}^{\mathcal{T}_{e \pm}}(\hat{p})=\left(\begin{array}{cc}
v \hat{p} & 0 \\
0 & -v \hat{p}
\end{array}\right)
$$

i.e., makes the Fermi velocities equal, $v_{+}=v_{-}=v$, and prohibits the Fermi-point mismatch, $k_{+}=k_{-}=k_{0}, \varepsilon_{0}=0$.

When combined with the CC operation $\mathcal{C}_{+}$[Eq. (2.6)], the TR operation $\mathcal{T}_{e \pm}$ [Eq. (A3)] for the electron part $\hat{\psi}_{e}(x)$ of the wave function naturally introduces the TR operation $\mathcal{T}_{h \pm}=$ $\hat{T}_{e \pm}^{*} \mathcal{K}$ for the hole part $\hat{\psi}_{h}(x)$. These electron $\mathcal{T}_{e \pm}$ and hole $\mathcal{T}_{h \pm}$ parts can be joined into the total TR operation

$$
\begin{aligned}
& \mathcal{T}_{ \pm}=\left(\begin{array}{cc}
\hat{T}_{e \pm} & \hat{0} \\
\hat{0} & a_{ \pm} \hat{T}_{e \pm}^{*}
\end{array}\right) \mathcal{K}, \\
& \hat{T}_{+}=\left(\begin{array}{cc}
\tau_{x} & \hat{0} \\
\hat{0} & a_{+} \tau_{x}
\end{array}\right), \quad \hat{T}_{-}=\left(\begin{array}{cc}
i \tau_{y} & \hat{0} \\
\hat{0} & a_{-} i \tau_{y}
\end{array}\right),
\end{aligned}
$$

acting on the full BdG wave function $\hat{\psi}(x)$ [Eq. (2.5)], where, importantly, $a_{ \pm}$is an arbitrary relative phase factor, $\left|a_{ \pm}\right|=1$.

Applying this TR operation to the Hamiltonian (2.11), we obtain that the most general form of the Hamiltonian satisfying the TR symmetry $\mathcal{T}_{ \pm}$[Eqs. (A2) and (A6)] has the form

$$
\hat{H}^{\mathcal{T}_{ \pm}}(\hat{p})=\left(\begin{array}{cccc}
v \hat{p} & 0 & 0 & \Delta(x) \\
0 & -v \hat{p} & -\Delta(x) & 0 \\
0 & -\Delta^{*}(x) & v \hat{p} & 0 \\
\Delta^{*}(x) & 0 & 0 & -v \hat{p}
\end{array}\right),
$$

where the pairing field $\Delta(x)$ and the relative phase factors $a_{ \pm}$ must satisfy the constraint

$$
\Delta(x)=\mp \Delta^{*}(x) a_{ \pm}^{*} .
$$


Hence, the pairing field must be a real function $\Delta^{\prime}(x)$, up to a constant phase factor,

$$
\Delta(x)=\Delta^{\prime}(x) e^{-i \delta},
$$

and the phase factors in Eq. (A6) must satisfy

$$
a_{ \pm}=\mp e^{2 i \delta} .
$$

In other words, to satisfy either of the symmetries $\mathcal{T}_{ \pm}$, the pairing field must be effectively real: the overall constant phase factor $e^{-i \delta}$ is physically inessential, since it can be adjusted by the phase factors of the basis functions of $\hat{\psi}_{e}(x)$ and $\hat{\psi}_{h}(x)$ parts, and so, it cannot affect the symmetry. The real function $\Delta^{\prime}(x)$ could, in principle, change sign, which would create a domain-wall structure.

In particular, under $\mathcal{T}_{ \pm}$symmetry, the one-harmonic coordinate dependence (4.1) of the pairing field is prohibited for finite momentum $q \neq 0$, which is in accord with the fact that under $\mathcal{T}_{ \pm}$the Fermi-point mismatch is also prohibited. This dependence was introduced in Sec. IV as a possible mechanism to mitigate the effect of the Fermi-point mismatch. When the latter is absent, there is no practical reason for this form of the pairing field. On the other hand, the constant pairing field $\Delta(x)=\Delta_{0}$ in the low-energy 1FS model is always $\mathcal{T}_{ \pm}$-symmetric.

We note an interesting property that if the low-energy Hamiltonian $\hat{H}(\hat{p})$ satisfies one of the $\mathcal{T}_{ \pm}$symmetries, i.e., Eqs. (A7) and (A9) hold, then it also automatically satisfies the other symmetry $\mathcal{T}_{\mp}$, respectively. In this sense, the other symmetry $\mathcal{T}_{\mp}$ has emergent character. At the same time, we also note that this symmetry relation is limited since, even though it holds for the bulk Hamiltonian, the properties of the BCs under $\mathcal{T}_{ \pm}$symmetries are radically different, as we show next.

\section{Time-reversal symmetries of the normal-reflection boundary conditions}

Now we turn to the TR symmetries $\mathcal{T}_{ \pm}$of the normalreflection BCs (3.5). As explained in Sec. III, the BCs satisfy a certain symmetry if the wave function transformed by the symmetry operation also satisfies those BCs. As with $\mathcal{C}_{+}$, TR operations $\mathcal{T}_{ \pm}$do not alter the coordinate and thus leave the geometry of the system intact; hence, the system with a boundary can, in principle, be $\mathcal{T}_{ \pm}$-symmetric. Inserting $\mathcal{T}_{ \pm} \hat{\psi}(0)$ [Eqs. (A1) and (A6)] into the normal-reflection BCs (3.5) with $v_{+}=v_{-}$, as required by $\mathcal{T}_{ \pm}$symmetry of the Hamiltonian (A7), we find that the BCs are $\mathcal{T}_{ \pm}$-symmetric if

$$
U_{e e}= \pm U_{e e},
$$

respectively.

Hence, the normal-reflection BCs (3.5) with $v_{+}=v_{-}$and arbitrary phase factor $U_{e e},\left|U_{e e}=1\right|$, satisfy $\mathcal{T}_{+}$symmetry.

On the other hand, interestingly, we obtain that there are no normal-reflection $B C s$ that satisfy $\mathcal{T}_{-}$symmetry. This result is the manifestation of the known physical effect: absence of backscattering between the counterpropagating 1D electron states of one Kramers pair $\psi_{e \pm}(x)$ (this effect is not related to superconductivity). One physical realization of such system with the low-energy Hamiltonian (A5) is the edge of a quantum spin Hall system in the topologically nontrivial phase [46]. The counterpropagating edge states go around the whole closed boundary of a 2D finite-size sample. Cutting the sample into parts will not cause backscattering; rather, the edge states will continue to propagate along the newly created edges of the parts. Thus, nonexistence of BCs for a $\mathcal{T}_{-}$-symmetric system of one Kramers pair with the Hamiltonian (A5) has a natural physical explanation and it is quite remarkable that the presented formalism of general BCs is "aware" of such physical effects.

In order to create an inaccessible region $x<0$ in such $\mathcal{T}_{-}$-symmetric quasi-1D system from which the states can backscatter, $\mathcal{T}_{-}$symmetry must necessarily be broken. In practice, this can be achieved by bringing a magnetic material in contact with the quantum-spin-Hall system (Fig. 2), which induces a gap in part of its edge, the situation we consider in Sec. VIII.

The BdG systems with $\mathrm{CC}$ symmetry $\mathcal{C}_{+}$and additional TR symmetries $\mathcal{T}_{ \pm}$belong to BDI and DIII symmetry classes, respectively. Note that the presence of $\mathcal{C}_{+}$and $\mathcal{T}_{ \pm}$symmetries automatically also generates chiral symmetries with the operations $\mathcal{T}_{ \pm} \mathcal{C}_{+}$. These, however, do not have a profound effect for the case of 1FS model.

As far as the consequences of the $\mathcal{T}_{ \pm}$symmetries for the Majorana bound states, we see that the additional $\mathcal{T}_{+}$symmetry does have an effect on the bulk, prohibiting the Fermi-point mismatch, but does not directly affect the Majorana bound state, which exists in the gapped superconducting state, regardless of whether $\mathcal{T}_{+}$is present or not. On the other hand, it is simply impossible to create a boundary without breaking the TR symmetry $\mathcal{T}_{-}$.

\section{Time-reversal symmetries of the generalized quantum-wire model}

We now consider the TR symmetries $\mathcal{T}_{ \pm}$of the generalized quantum-wire model of Sec. VII and establish the relation between them and those of the low-energy model, to which it reduces.

The actual TR symmetry $\mathcal{T}_{e-}=i \sigma_{y} \mathcal{K}$ of electrons with the Hamiltonian $\hat{\mathcal{H}}_{e}(\hat{k})$ [Eq. (7.2)] is broken by the Zeeman field, which transforms as

$$
\mathcal{T}_{e-}:\left(h_{x}, h_{y}, h_{z}\right) \rightarrow-\left(h_{x}, h_{y}, h_{z}\right) .
$$

However, as was noticed in Refs. [45,47,48], the electron Hamiltonian $\hat{\mathcal{H}}_{e 0}(\hat{k})$ [Eq. (7.3)] with only the $h_{\perp}$ Zeeman field possesses an effective TR symmetry $\mathcal{T}_{e+}=\sigma_{x} \mathcal{K}$. This TR operation arises as the product $\mathcal{T}_{e+}=\Sigma_{z} \mathcal{T}_{e-}$ of the actual TR operation $\mathcal{T}_{e-}$ and the reflection $\Sigma_{z}$ along the horizontal direction perpendicular to the wire, Fig. 1. The Zeeman field transforms as

$$
\Sigma_{z}:\left(h_{x}, h_{y}, h_{z}\right) \rightarrow\left(-h_{x}-h_{y}, h_{z}\right)
$$

under the latter and, hence, transforms as

$$
\mathcal{T}_{e+}:\left(h_{x}, h_{y}, h_{z}\right) \rightarrow\left(h_{x}, h_{y},-h_{z}\right)
$$

under the effective $\mathcal{T}_{e+}$. Thus, the components $h_{x, y}$ of the Zeeman field in the vertical $x y$ plane containing the wire are preserved under $\mathcal{T}_{e+}$, even though they are not preserved under either of these two operations individually. Importantly, the 
electron system with a boundary satisfies $\mathcal{T}_{e+}$, and indeed, the hard-wall BCs (7.18) satisfy $\mathcal{T}_{e+}$.

The effective TR symmetry $\mathcal{T}_{e+}$ of the electron part of the microscopic quantum-wire model [Eqs. (7.3) and (7.18)] with $h_{z}=0$ translates to that [Eq. (A3)] of the low-energy model, in accord with Appendix A 1: it prohibits the Fermipoint mismatch $\left[\varepsilon_{0}=0, k_{+}=k_{-}=k_{0}\right.$, Eq. (7.15)], enforces equal velocities $\left[v_{+}=v_{-}=v\right.$, Eq. (7.14)], but provides no constraints on the scattering phase factor $U_{e e}$ [Eq. (7.22)] of the normal-reflection BCs (3.5).

Further, as for the low-energy model (Appendix A 1), the general forms of the TR operations for the BdG Hamiltonian (7.9) of the wire read

$$
\begin{aligned}
& \mathcal{T}_{ \pm}=\left(\begin{array}{cc}
\hat{T}_{e \pm} & \hat{0} \\
\hat{0} & \tilde{a}_{ \pm} \hat{T}_{e \pm}^{*}
\end{array}\right) \mathcal{K}, \\
& \hat{T}_{+}=\left(\begin{array}{cc}
\tau_{x} & \hat{0} \\
\hat{0} & \tilde{a}_{+} \tau_{x}
\end{array}\right), \quad \hat{T}_{-}=\left(\begin{array}{cc}
i \tau_{y} & \hat{0} \\
\hat{0} & \tilde{a}_{-} i \tau_{y}
\end{array}\right),
\end{aligned}
$$

with adjustable phase factors $\tilde{a}_{ \pm},\left|\tilde{a}_{ \pm}\right|=1$. Applying these operations, we find that the pairing field (7.11) is $\mathcal{T}_{+}$symmetric if its components and $\tilde{a}_{+}$satisfy

$$
\Delta_{0, z}(x ; k)=-\tilde{a}_{+}^{*} \Delta_{0, z}^{*}(X, k), \quad \Delta_{x, y}(x ; k)=\tilde{a}_{+}^{*} \Delta_{x, y}^{*}(X, k)
$$

(note that different triplet components transform differently; this is in accord with $\mathcal{T}_{e+}=\mathcal{T}_{e-} \Sigma_{z}$ involving a spatial operation, so the spin orientation of the pairing field does matter). These relations are satisfied when

$$
\begin{aligned}
\Delta_{0, z}(x ; k) & =e^{-i \delta} \Delta_{0, z}^{\prime}(x ; k), \quad \Delta_{x, y}(x ; k)=e^{-i \delta} i \Delta_{x, y}^{\prime}(x ; k), \\
e^{2 i \delta} & =-\tilde{a}_{+},
\end{aligned}
$$

where $\Delta_{0, x, y, z}^{\prime}(x ; k)$ are real. Inserting this form into Eq. (7.16) for the low-energy pairing field $\Delta(x)$, we find that the latter does have the form (A9) required for it to be $\mathcal{T}_{+}$-symmetric.

Similarly, the pairing field is $\mathcal{T}_{-}$-symmetric when

$$
\Delta_{0, x, y, z}(x ; k)=\tilde{a}_{-}^{*} \Delta_{0, x, y, z}^{*}(x ; k) .
$$

This holds when

$$
\Delta_{0, x, y, z}(x ; k)=e^{-i \delta} \Delta_{0, x, y, z}^{\prime}(x ; k), \quad e^{2 i \delta}=\tilde{a}_{-},
$$

where $\Delta_{0, x, y, z}^{\prime}(x ; k)$ are real. Inserting this form into Eq. (7.16) for $\Delta(x)$, we find that the latter does not generally have the form (A9) required for it to be $\mathcal{T}_{-}$-symmetric. This is not surprising since, even if the microscopic pairing field is $\mathcal{T}_{-}$symmetric, the electron Hamiltonian $\hat{\mathcal{H}}_{e 0}(\hat{k})$ [Eq. (7.3)] is not, and it is involved in the low-energy projection (7.16).

These results and those of Appendix A 1 illustrate the general property that the symmetries of the microscopic model and of the low-energy model to which the former reduces are not necessarily identical. If the microscopic model possesses some symmetries, then surely the low-energy model does also, which is the case for $\mathcal{T}_{+}$symmetry here. However, the low-energy model can possess some additional, emergent symmetries that are not present in the microscopic model from which it originates. This is the case for $\mathcal{T}_{-}$symmetry here (and this applies only to the low-energy bulk Hamiltonian, but not to the BCs). At the end of Appendix A 1, we noted that if the low-energy BdG Hamiltonian satisfies $\mathcal{T}_{+}$symmetry

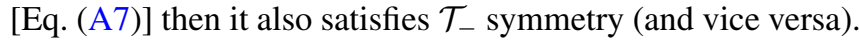
However, in the microscopic model, $\mathcal{T}_{-}$symmetry is broken.
[1] A. Y. Kitaev, Phys. Usp. 44, 131 (2001).

[2] L. Fu and C. L. Kane, Phys. Rev. B 79, 161408(R) (2009).

[3] R. M. Lutchyn, J. D. Sau, and S. Das Sarma, Phys. Rev. Lett. 105, 077001 (2010).

[4] Y. Oreg, G. Refael, and F. von Oppen, Phys. Rev. Lett. 105, 177002 (2010).

[5] A. C. Potter and P. A. Lee, Phys. Rev. Lett. 105, 227003 (2010).

[6] A. R. Akhmerov, Phys. Rev. B 82, 020509(R) (2010).

[7] M. Wimmer, A. R. Akhmerov, M. V. Medvedyeva, J. Tworzydło, and C. W. J. Beenakker, Phys. Rev. Lett. 105, 046803 (2010).

[8] A. R. Akhmerov, J. P. Dahlhaus, F. Hassler, M. Wimmer, and C. W. J. Beenakker, Phys. Rev. Lett. 106, 057001 (2011).

[9] R. M. Lutchyn, T. D. Stanescu, and S. Das Sarma, Phys. Rev. Lett. 106, 127001 (2011).

[10] I. C. Fulga, F. Hassler, A. R. Akhmerov, and C. W. J. Beenakker, Phys. Rev. B 83, 155429 (2011).

[11] T. D. Stanescu, R. M. Lutchyn, and S. Das Sarma, Phys. Rev. B 84, 144522 (2011).

[12] A. C. Potter and P. A. Lee, Phys. Rev. B 83, 094525 (2011).

[13] B. Zhou and S.-Q. Shen, Phys. Rev. B 84, 054532 (2011).

[14] K. T. Law and P. A. Lee, Phys. Rev. B 84, 081304(R) (2011).

[15] R. M. Lutchyn and M. P. A. Fisher, Phys. Rev. B 84, 214528 (2011).
[16] K. Yada, M. Sato, Y. Tanaka, and T. Yokoyama, Phys. Rev. B 83, 064505 (2011).

[17] M. Sato, Y. Tanaka, K. Yada, and T. Yokoyama, Phys. Rev. B 83, 224511 (2011).

[18] G. Kells, D. Meidan, and P. W. Brouwer, Phys. Rev. B 85, 060507(R) (2012).

[19] M. Gibertini, F. Taddei, M. Polini, and R. Fazio, Phys. Rev. B 85, 144525 (2012).

[20] S. Rex and A. Sudbø, Phys. Rev. B 90, 115429 (2014).

[21] F. Crépin, B. Trauzettel, and F. Dolcini, Phys. Rev. B 89, 205115 (2014).

[22] S. Ikegaya, S.-I. Suzuki, Y. Tanaka, and Y. Asano, Phys. Rev. B 94, 054512 (2016).

[23] J. Alicea, Rep. Prog. Phys. 75, 076501 (2012).

[24] M. Leijnse and K. Flensberg, Semicond. Sci. Technol. 27, 124003 (2012).

[25] Y. Tanaka, M. Sato, and N. Nagaosa, J. Phys. Soc. Japan 81, 011013 (2012).

[26] C. W. J. Beenakker, Annu. Rev. Condens. Matter Phys. 4, 113 (2013).

[27] V. Mourik, K. Zuo, S. M. Frolov, S. R. Plissard, E. P. A. M. Bakkers, and L. P. Kouwenhoven, Science 336, 1003 (2012).

[28] H. O. H. Churchill, V. Fatemi, K. Grove-Rasmussen, M. T. Deng, P. Caroff, H. Q. Xu, and C. M. Marcus, Phys. Rev. B 87, 241401(R) (2013). 
[29] B. Jäck, Y. Xie, J. Li, S. Jeon, B. A. Bernevig, and A. Yazdani, Science 364, 1255 (2019).

[30] P.-G. de Gennes, Superconductivity of Metals and Alloys (Benjamin, New York, 1966).

[31] C.-K. Chiu, J. C. Y. Teo, A. P. Schnyder, and S. Ryu, Rev. Mod. Phys. 88, 035005 (2016).

[32] S. Ryu, A. Schnyder, A. Furusaki, and A. Ludwig, New J. Phys. 12, 065010 (2010).

[33] M. V. Berry and R. J. Mondragon, Proc. R. Soc. Lond. A 412, 53 (1987).

[34] G. Bonneau, J. Faraut, and G. Valent, Am. J. Phys. 69, 322 (2001).

[35] I. V. Tokatly, A. G. Tsibizov, and A. A. Gorbatsevich, Phys. Rev. B 65, 165328 (2002).

[36] E. McCann and V. I. Fal'ko, J. Phys.: Condens. Matter 16, 2371 (2004).

[37] A. R. Akhmerov and C. W. J. Beenakker, Phys. Rev. Lett. 98, 157003 (2007).

[38] A. R. Akhmerov and C. W. J. Beenakker, Phys. Rev. B 77, 085423 (2008).

[39] J. A. M. van Ostaay, A. R. Akhmerov, C. W. J. Beenakker, and M. Wimmer, Phys. Rev. B 84, 195434 (2011).

[40] M. T. Ahari, G. Ortiz, and B. Seradjeh, Am. J. Phys. 84, 858 (2016).

[41] M. Kharitonov, J.-B. Mayer, and E. M. Hankiewicz, Phys. Rev. Lett. 119, 266402 (2017).

[42] D. R. Candido, M. Kharitonov, J. C. Egues, and E. M. Hankiewicz, Phys. Rev. B 98, 161111(R) (2018).

[43] B. Seradjeh and M. Vennettilli, Phys. Rev. B 97, 075132 (2018).

[44] K. N. Nesterov, Manuel Houzet, and Julia S. Meyer, Phys. Rev. B 93, 174502 (2016).
[45] K. V. Samokhin, Phys. Rev. B 101, 094502 (2020).

[46] B. A. Bernevig, T. L. Hughes, and S. C. Zhang, Science 314, 1757 (2006).

[47] K. V. Samokhin, Phys. Rev. B 95, 064504 (2017).

[48] K. V. Samokhin, Ann. Phys. (NY) 385, 563 (2017).

[49] $\mathcal{T}_{e \pm}$ with the label $e$ will denote the symmetries of just the electron system, whereas $\mathcal{T}_{ \pm}$will denote the symmetries of the whole BdG system of electrons and holes, in the presence of superconductivity. This distinction is useful, since the superconducting pairing field could, in principle, break these symmetries; see discussion in Appendix.

[50] A. I. Larkin and Yu. N. Ovchinnikov, Zh. Eksp. Teor. Fiz. 47, 1136 (1964) [Sov. Phys. JETP 20, 762 (1965)].

[51] P. Fulde and R. A. Ferrell, Phys. Rev. 135, A550 (1964).

[52] We will refer to these models as "microscopic" in relation to the derived low-energy model in the sense that they contain more degrees of freedom or the structure of their boundary is specified. These models, however, could themselves arise as low-energy models from other, "more microscopic" models.

[53] Note that the Fermi points $\pm k_{0}$ [Eq. (7.7)] of the Hamiltonian $\hat{\mathcal{H}}_{e 0}(\hat{k})$ without $h_{z}$ are actually those symmetric crossing points (2.1) of the electron and hole normal-state bands in the presence of (small) $h_{z}$; compare Figs. 1 and 3.

[54] We mention that for the continuum quantum-wire model one could, in principle, also derive and study the most general form of the BCs; here, we consider the familiar hard-wall BCs as an example, which are just one instance of the latter.

[55] M. Rouco, I. V. Tokatly, and F. S. Bergeret, Phys. Rev. B 99, 094514 (2019).

[56] M. Rouco, F. S. Bergeret, and I. V. Tokatly, Phys. Rev. B 103, 064505 (2021). 